\title{
Sr isotopic characteristics in two small watersheds draining silicate and carbonate rocks: implication for studies on seawater $\mathrm{Sr}$ isotopic evolution
}

\author{
W. H. Wu ${ }^{1}$, H. B. Zheng ${ }^{2}$, J. H. Cao ${ }^{3}$, and J. D. Yang ${ }^{4}$ \\ ${ }^{1}$ Key Laboratory of Surficial Geochemistry, Ministry of Education; School of Earth Sciences and Engineering, \\ Nanjing University, Nanjing, 210093, China \\ ${ }^{2}$ School of Geography Science, Nanjing Normal University, Nanjing, 210046, China \\ ${ }^{3}$ Institute of Karst Geology, Chinese Academy of Geological Science, Guilin, 541004, China \\ ${ }^{4}$ Center of Modern Analysis, Nanjing University, Nanjing, 210093, China \\ Correspondence to: W. H. Wu (wuwh@nju.edu.cn)
}

Received: 30 May 2013 - Published in Hydrol. Earth Syst. Sci. Discuss.: 21 June 2013

Revised: 23 November 2013 - Accepted: 31 December 2013 - Published: 13 February 2014

\begin{abstract}
We systematically investigated the $\mathrm{Sr}$ isotopic characteristics of a small silicate watershed, the Xishui River a tributary of the Yangtze River, and a small carbonate watershed, the Guijiang River a tributary of the Pearl River. The results show that the two rivers have uncommon $\mathrm{Sr}$ isotopic characteristics compared with most small watersheds. Specifically, the silicate watershed (Xishui River) has relatively high $\mathrm{Sr}$ concentrations $\left(0.468\right.$ to $1.70 \mu \mathrm{mol} \mathrm{L}^{-1}$ in summer and 1.30 to $3.17 \mu \mathrm{mol} \mathrm{L} \mathrm{L}^{-1}$ in winter, respectively) and low ${ }^{87} \mathrm{Sr} /{ }^{86} \mathrm{Sr}$ ratios $(0.708686$ to 0.709148 in summer and 0.708515 to 0.709305 in winter). The carbonate watershed (Guijiang River) has low $\mathrm{Sr}$ concentrations $(0.124$ to $\left.1.098 \mu \mathrm{mol} \mathrm{L}{ }^{-1}\right)$ and high ${ }^{87} \mathrm{Sr} /{ }^{86} \mathrm{Sr}$ ratios $(0.710558$ to $0.724605)$.

As the ${ }^{87} \mathrm{Sr} /{ }^{86} \mathrm{Sr}$ ratios in the Xishui River are lower than those in seawater, the ${ }^{87} \mathrm{Sr} /{ }^{86} \mathrm{Sr}$ ratio of seawater will decrease after the river water is transported to the oceans. Previous studies have also shown that some basaltic watersheds with extremely high chemical weathering rates reduced the seawater Sr isotope ratios. In other words, river catchments with high silicate weathering rates do not certainly transport highly radiogenic $\mathrm{Sr}$ into oceans. Therefore, the use of the variations in the seawater ${ }^{87} \mathrm{Sr} /{ }^{86} \mathrm{Sr}$ ratio to indicate the continental silicate weathering intensity may be questionable.

In the Guijiang River catchment, the ${ }^{87} \mathrm{Sr} /{ }^{86} \mathrm{Sr}$ ratios of carbonate rocks and other sources (rainwater, domestic and industrial waste water, and agricultural fertilizer) are lower
\end{abstract}

than 0.71. In comparison, some non-carbonate components, such as sand rocks, mud rocks, and shales, have relatively high $\mathrm{Sr}$ isotopic compositions. Moreover, granites accounted for only $5 \%$ of the drainage area have extremely high ${ }^{87} \mathrm{Sr} /{ }^{86} \mathrm{Sr}$ ratios with an average of greater than 0.8 . Therefore, a few silicate components in carbonate rocks obviously increase the Sr isotopic compositions of the river water.

\section{Introduction}

By analyzing the $\mathrm{Sr}$ isotopic compositions of marine limestones and their shells, the evolution curve of the seawater ${ }^{87} \mathrm{Sr} /{ }^{86} \mathrm{Sr}$ ratio in the Phanerozoic was well established (Veizer and Compston, 1974; Brass, 1976; Burke et al., 1982; Elderfield and Gieskes, 1982; DePaolo and Ingram, 1985; Palmer and Elderfield, 1985; DePaolo, 1986; Hess et al., 1986; Richter and DePaolo, 1987, 1988; Raymo et al., 1988; Veizer, 1989; Capo and DePaolo, 1990; Hodell et al., 1991; Richter et al., 1992; Veizer et al., 1999; Korte et al., 2006; Melezhik et al., 2009). The Sr isotope budget of the oceans is dominated by its supplies via rivers, hydrothermal vent waters, and diagenesis of deep-sea sediments, and the dissolved Sr flux to the modern oceans via rivers is far more than those via the latter two (Palmer and Edmond, 1989). In particular, the Ganges and Brahmaputra, originating in the southern Qinghai-Tibet Plateau, are characterized by both high $\mathrm{Sr}$ 
concentrations and high ${ }^{87} \mathrm{Sr} /{ }^{86} \mathrm{Sr}$ ratios and thus have a major influence on the increase in the $\mathrm{Sr}$ isotopic composition of seawater (e.g., Krishnaswami et al., 1992; Harris, 1995; Derry and France-Lanord, 1996; Quade et al., 1997; Singh et al., 1998, 2006; Galy et al., 1999; English et al., 2000; Dalai et al., 2003). Therefore, many researchers attribute the steady increase in the ${ }^{87} \mathrm{Sr} /{ }^{86} \mathrm{Sr}$ ratio in the oceans since the Cenozoic mainly to the uplifting of the Qinghai-Tibet Plateau, which caused increased silicate weathering and highly radiogenic Sr flux to the oceans. As the silicate rocks in the southern Himalayas have both high ${ }^{87} \mathrm{Sr} /{ }^{86} \mathrm{Sr}$ ratios and high $\mathrm{Sr}$ concentrations, the rise of the $\mathrm{Sr}$ isotopic composition of seawater since the Cenozoic can be used as a proxy of intensified silicate weathering (Palmer and Elderfield, 1985; Raymo et al., 1988; Edmond, 1992; Krishnaswami et al., 1992; Richter et al., 1992; Harris, 1995; Blum, 1997; Galy et al., 1999; Chesley et al., 2000; English et al., 2000; Bickle et al., 2005). However, other studies have shown that the unusual metamorphic evolution of the Himalayas was enriched with carbonates, resulting in abnormally high ${ }^{87} \mathrm{Sr} /{ }^{86} \mathrm{Sr}$ ratios, and that the weathering of such carbonates might control the ${ }^{87} \mathrm{Sr} /{ }^{86} \mathrm{Sr}$ ratios of river water (e.g., Edmond, 1992; Palmer and Edmond, 1992; Quade et al., 1997; Blum et al., 1998; Harris et al., 1998; English et al., 2000; Karim and Veizer, 2000; Jacobson and Blum, 2000; Bickle et al., 2001; Jacobson et al., 2002a). If much of the radiogenic Sr in the rivers is derived from carbonate weathering, then changes in the seawater Sr isotopic composition would not be a proxy of the continental silicate weathering intensity.

To better understand the contribution of silicate and carbonate weathering to the $\mathrm{Sr}$ isotopic composition of river water, we selected two small watersheds: one was the Xishui River draining silicate rocks, a tributary of the Yangtze River, and another was the Guijiang River draining carbonate rocks, a tributary of the Pearl River. In the two river catchments, silicate and carbonate rocks account for approximately $95 \%$ of the respective drainage area. By analyzing the $\mathrm{Sr}$ isotopic compositions of river waters, we will discuss the Sr isotope characteristics and their controlling factors in small watersheds and investigate the relationship between silicate, carbonate weathering, and the $\mathrm{Sr}$ isotope evolution of seawater.

\section{Studied areas}

The Xishui River is a small tributary of the Yangtze River located at $115^{\circ} 07^{\prime}-116^{\circ} 05^{\prime} \mathrm{E}$ and $30^{\circ} 20^{\prime}-31^{\circ} 09^{\prime} \mathrm{N}$. This river originates from south of the Dabie Mountain (elevation $1600 \mathrm{~m}$ ), has a length of $157 \mathrm{~km}$ and a drainage area of $2670 \mathrm{~km}^{2}$. Its headwater is composed of the Donghe River and Xihe River, which converge in Yishan County. The Xishui River merges into the Yangtze River at Lanxi (Fig. 1). The Xishui River catchment belongs to a subtropical monsoon climate with a mean temperature of $29^{\circ} \mathrm{C}$ in the summer and $4^{\circ} \mathrm{C}$ in the winter and an annual mean rainfall

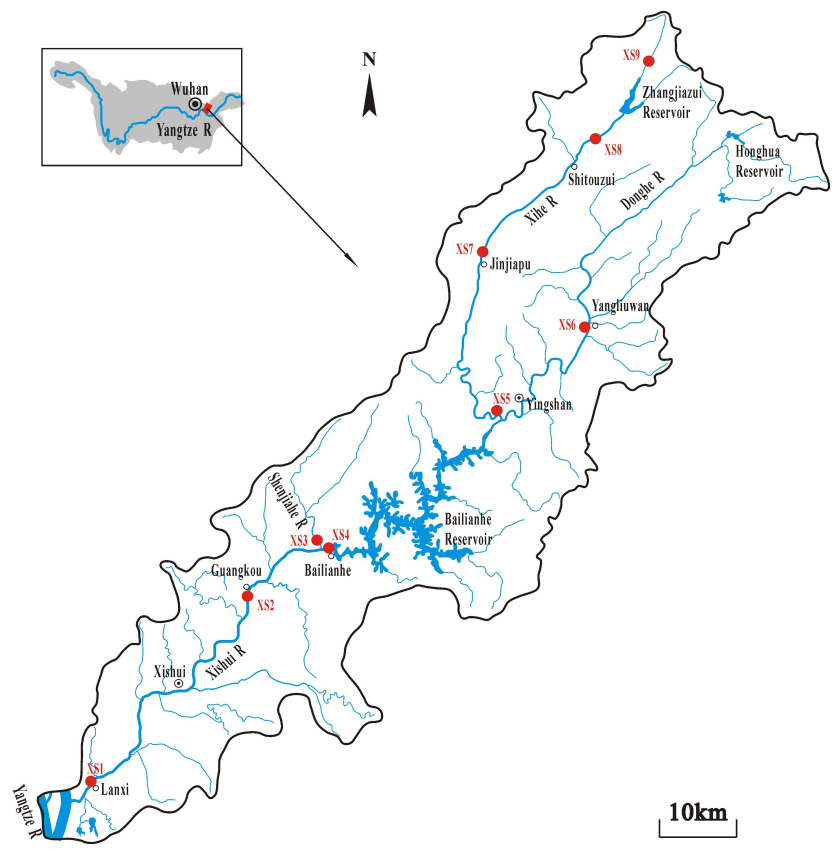

Fig. 1. Map of the Xishui River catchment and sampling locations (filled red circles).

of approximately $1350 \mathrm{~mm}$. The Xishui River mainly flows across the Dabie Mountain early Proterozoic metamorphic zone, which is altered by multiphase tectonism and regional metamorphism, forming a variety of metamorphic rocks and complete metamorphic facies. The catchment is covered with ultrahigh-pressure metamorphic rocks, mainly composed of eclogite, gneiss, and some granite (Fig. 2) (Bureau of Geology and Mineral Resources of Hubei Province, 1990; China Geological Survey, 2004).

The Guijiang River, a tributary of the Pearl River, rises in the Miaoershan Mountain with an elevation of $2142 \mathrm{~m}$. This river has a length of $438 \mathrm{~km}$ and a drainage area of $18790 \mathrm{~km}^{2}$. The upper reaches are upstream of Rongjiang Town, and the middle reaches are from the confluence with the Lingqu River to Pingle County, also called "the Lijiang River", and then merge into the Pearl River at Wuzhou City (Fig. 3). The annual rainfall and evaporation are approximately $2000 \mathrm{~mm}$ and 1100 to $1200 \mathrm{~mm}$, respectively, and the annual average temperature is $\sim 20^{\circ} \mathrm{C}$ in the Guijiang River catchment. Silurian granites, Ordovician-Cambrian shales, and mud rocks intercalate carbonate rocks and are mainly exposed in the headwater and upper reaches. The middle reaches are almost entirely covered with Devonian carbonate rocks, and the lower reaches flow across Cambrian terrain composed largely of carbonate rocks in intercalated shales (Fig. 4) (China Geological Survey, 2004). 


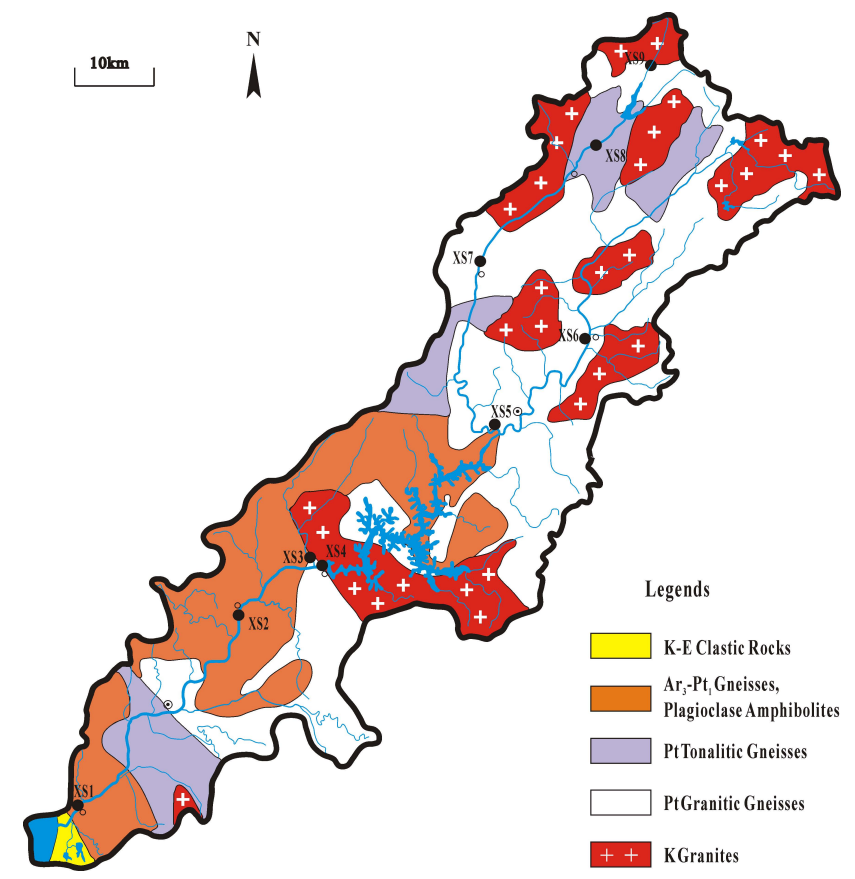

Fig. 2. Geological map of the Xishui River catchment (modified from the China Geological Survey, 2004).

\section{Sampling and analysis}

From the river mouths to the source areas of the Xishui River and Guijiang River, 57 samples of river water, 1 sample of rain water, 1 sample of snow, 27 samples of riverbed sediment, and 2 samples of soil were collected in July 2010, December 2010, and July 2011 (Figs. 1 and 3, Table 1). A portable water quality analyzer was used to measure the temperature, $\mathrm{pH}$, and conductivity in situ. Flow measurement was used to measure the flow velocity in the field and to estimate the water discharge. All of the samples were collected from the river bank or midstream away from towns, avoiding contamination from anthropogenic activities, and were stored in pre-cleaned polyethylene bottles free of air. The water samples were filtered through a $0.45 \mu \mathrm{m}$ mixed cellulose esters Millipore filter. An aliquot of the filtered water was acidified to $\mathrm{pH}<2$ with ultrapure grade 1:1 nitric acid. $\mathrm{Ca}^{2+}, \mathrm{Mg}^{2+}, \mathrm{Na}^{+}, \mathrm{K}^{+}, \mathrm{Sr}$, and $\mathrm{Si}$ were measured in the filtered and acidified water using an inductively coupled plasma spectrometer (ICP-AES, JarrellAsh1100) at the Center of Modern Analysis of Nanjing University. The anions $\left(\mathrm{F}^{-}, \mathrm{Cl}^{-}, \mathrm{NO}_{3}^{-}\right.$, and $\left.\mathrm{SO}_{4}^{2-}\right)$ in the filtered and un-acidified samples were measured using an ion chromatograph (Dionex series 1100) at the Key Laboratory of Surficial Geochemistry, Ministry of Education, School of Earth Sciences and Engineering, Nanjing University. The alkalinity was measured in the unfiltered water samples using a digital titrator (Hach 16900). The measurement reproducibility was determined by repeat analyses of samples

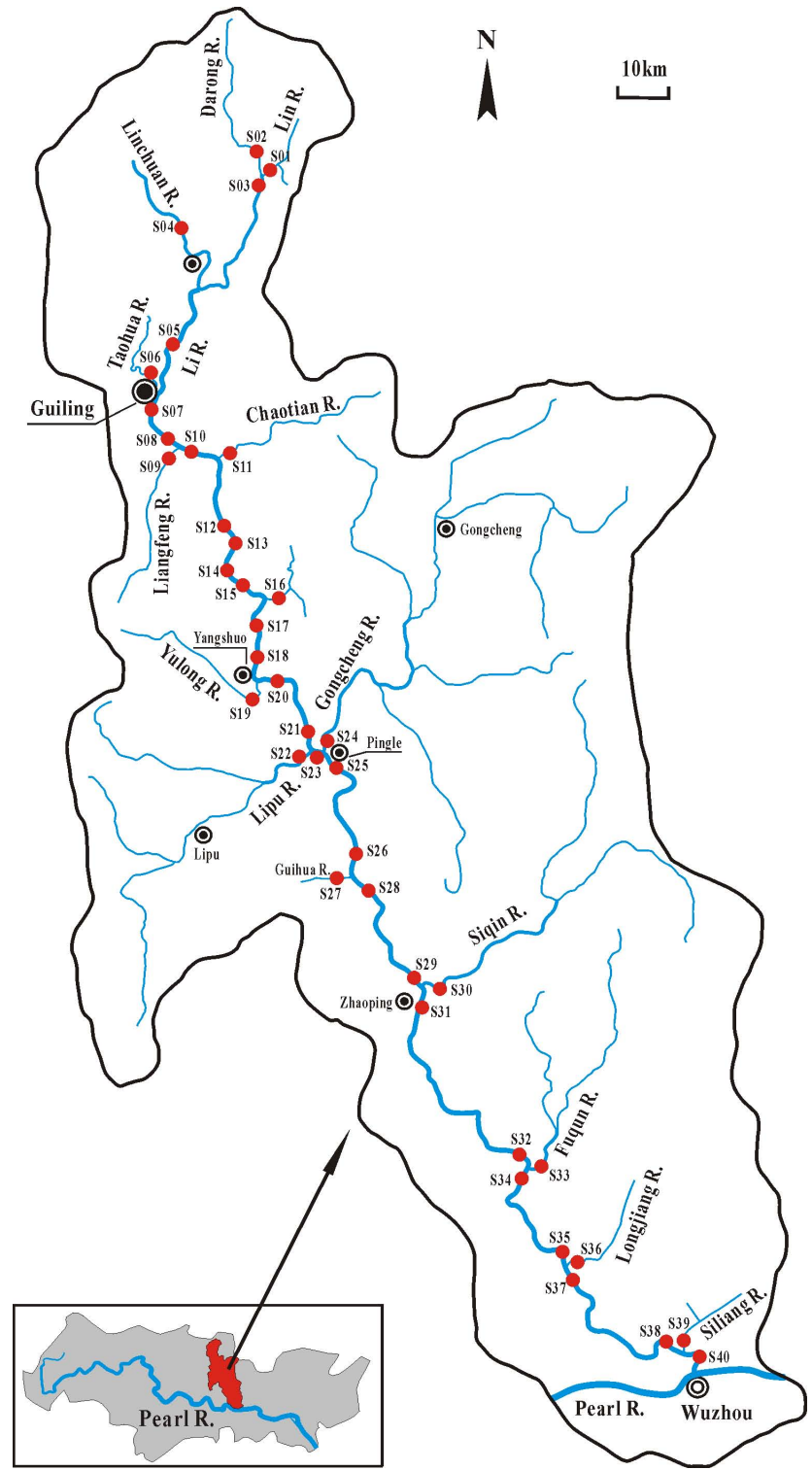

Fig. 3. Map of the Guijiang River catchment and sampling locations (filled red circles).

and standards, which showed $\pm 2 \%$ precision for the cations and $\pm 5 \%$ for the anions. For most of the water samples, the total cation charge $\left(\mathrm{TZ}^{+}=\mathrm{Ca}^{2+}+\mathrm{Mg}^{2+}+\mathrm{Na}^{+}+\mathrm{K}^{+}\right.$ in meq $\mathrm{L}^{-1}$ ) balanced the total anion charge $\left(\mathrm{TZ}^{-}=\mathrm{HCO}_{3}^{-}+\mathrm{Cl}^{-}+\mathrm{SO}_{4}^{2-}\right.$ in $\left.\mathrm{meqL}^{-1}\right)$ within the analytical uncertainties, and the normalized inorganic charge balance $\left(\mathrm{NICB}=\left(\mathrm{TZ}^{+}-\mathrm{TZ}^{-}\right) / \mathrm{TZ}^{+} \times 100 \%\right)$ was within $\pm 5 \%$ (Wu et al., 2013). For the $\mathrm{Sr}$ isotope ratio analysis, the Sr was separated from the samples using standard ion exchange techniques. The $\mathrm{Sr}$ isotopic compositions were measured using a Finnigan Triton thermal ionization mass spectrometer at the State Key Laboratory for Mineral Deposits Research, Nanjing University. The reproducibility and accuracy of the $\mathrm{Sr}$ isotope runs were periodically checked by 


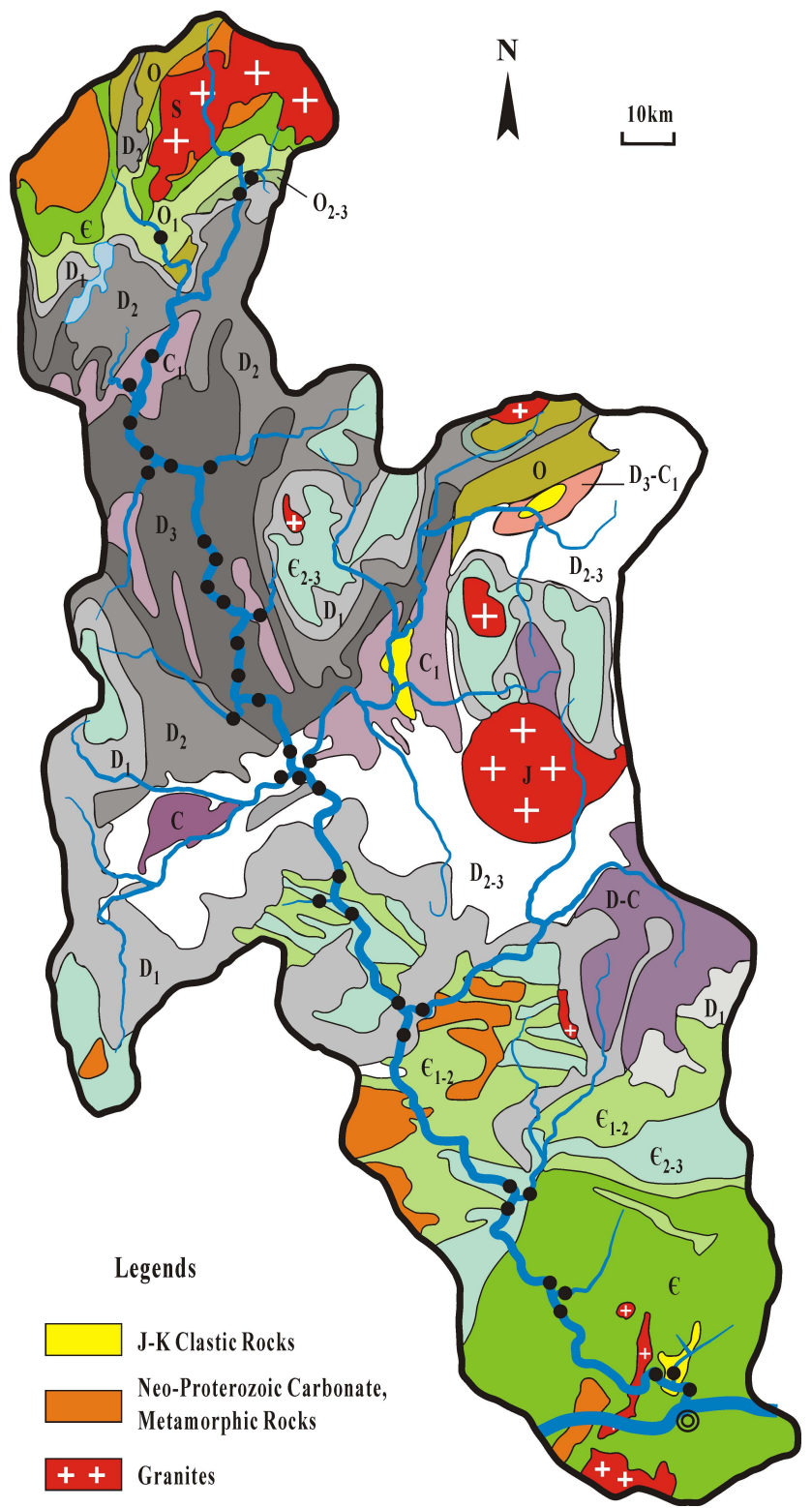

Fig. 4. Geological map of the Guijiang River catchment (modified from the China Geological Survey, 2004). $\epsilon$ : Cambrian mixed layer; $\epsilon_{1-2}$ : limestones/dolomites intercalated shales; $\epsilon_{2-3}$ : limestones intercalated shales; $O$ : Ordovician mixed layer; $\mathrm{O}_{1}$ : shales, mud rocks, limestones, and dolomites; $D_{1}$ : sand rocks, mud rocks, and a few carbonate rocks; $D_{2}$ : carbonate rocks and detrital rocks; $D_{2-3}$ : carbonate rocks, sand rocks, and mud rocks; $D_{3}$ : carbonate rocks; $D_{3}-C_{1}$ : detrital rocks, carbonate rocks, and mud rocks; $D-$ $C$ : Devonian-Carboniferous mixed layer; $C$ : Carboniferous mixed layer; $C_{1}$ : carbonate rocks, sand rocks, and mud rocks intercalated coals.

running the Standard Reference Material NBS 987, with a mean ${ }^{87} \mathrm{Sr} /{ }^{86} \mathrm{Sr}$ ratio of $0.710248 \pm 20(2 \sigma$ external standard deviation, $n=15)$. The $\mathrm{Sr}$ isotopic ratios were normalized to ${ }^{86} \mathrm{Sr} /{ }^{88} \mathrm{Sr}=0.1194$. The analytical blank was $<1 \mathrm{ng}$ for $\mathrm{Sr}$.
Only the $<63 \mu \mathrm{m}$ fine-grained fractions of the riverbed sediment and soil samples were used. The calcite in the samples was selectively dissolved with purified acetic acid $\left(0.5 \mathrm{~mol} \mathrm{~L}^{-1}\right)$ at room temperature for up to $8 \mathrm{~h}$, and only the silicate fractions were investigated. All of the pretreated samples were cleaned in pure water, powdered in an agate mill, and then digested with a mixture of $\mathrm{HCl}+\mathrm{HNO}_{3}+\mathrm{HClO}_{4}+\mathrm{HF}$. The analysis of $\mathrm{Sr}$ concentrations and isotopic compositions is the same as for the water samples.

\section{Results}

The Sr concentrations and ${ }^{87} \mathrm{Sr} /{ }^{86} \mathrm{Sr}$ ratios of the water samples in the Xishui and Guijiang rivers are listed in Table 2. The Sr concentrations of the Xishui River are 0.468 to $1.70 \mu \mathrm{mol} \mathrm{L}^{-1}$ in the summer and 1.30 to $3.17 \mu \mathrm{mol} \mathrm{L}^{-1}$ in the winter, reflecting a dilution effect from high runoff in the summer. The ${ }^{87} \mathrm{Sr} /{ }^{86} \mathrm{Sr}$ ratios range from 0.708686 to 0.709148 in the summer and from 0.708515 to 0.709305 in the winter, and do not exhibit obvious seasonal variations. A snow sample from Lanxi has a $\mathrm{Sr}$ concentration of $0.879 \mu \mathrm{mol} \mathrm{L}^{-1}$ and a ${ }^{87} \mathrm{Sr} /{ }^{86} \mathrm{Sr}$ ratio of 0.709495 . The $\mathrm{Sr}$ concentrations are 0.124 to $1.098 \mu \mathrm{mol} \mathrm{L}{ }^{-1}$ and ${ }^{87} \mathrm{Sr} /{ }^{86} \mathrm{Sr}$ ratios are 0.710558 to 0.724605 in the Guijiang River. The rainwater sample from Zhaoping has a $\mathrm{Sr}$ concentration of $0.11 \mu \mathrm{mol} \mathrm{L}{ }^{-1}$ and a ${ }^{87} \mathrm{Sr} /{ }^{86} \mathrm{Sr}$ ratio of 0.710416 . Compared with the Xishui River, the Guijiang River has lower Sr concentrations but higher ${ }^{87} \mathrm{Sr} /{ }^{86} \mathrm{Sr}$ ratios. A plot of ${ }^{87} \mathrm{Sr} /{ }^{86} \mathrm{Sr}$ vs. $1 / \mathrm{Sr}$ shows that the silicate watershed Xishui River is characterized by carbonate weathering with low ${ }^{87} \mathrm{Sr} /{ }^{86} \mathrm{Sr}$ ratios and high $\mathrm{Sr}$ concentrations, and the carbonate watershed Guijiang River is closer to the silicate endmember with high ${ }^{87} \mathrm{Sr} /{ }^{86} \mathrm{Sr}$ ratios and low $\mathrm{Sr}$ concentrations (Fig. 5). It is surprising that each of the rivers exhibits entirely opposite $\mathrm{Sr}$ isotope characteristics than those of classic silicate and carbonate weathering. These reasons will be analyzed in Sect. 5 .

Table 2 also contains some major ion concentrations in the Xishui River (Wu et al., 2013). For the Guijiang River, we did not analyze major ions, and some data from other researchers are listed in Table 2 for reference.

The $\mathrm{Sr}$ characteristics of the riverbed sediments and soils are given in Table 3. The ${ }^{87} \mathrm{Sr} /{ }^{86} \mathrm{Sr}$ ratios of the Xishui riverbed sediments are 0.707058 to 0.712616 . The Guijiang riverbed sediments have ${ }^{87} \mathrm{Sr} /{ }^{86} \mathrm{Sr}$ ratios ranging from 0.735172 to 0.775952 , and two soil samples GJ15 and $\mathrm{GJ} 19$, have ${ }^{87} \mathrm{Sr} /{ }^{86} \mathrm{Sr}$ ratios of 0.744095 and 0.749902 , respectively. The ${ }^{87} \mathrm{Sr} /{ }^{86} \mathrm{Sr}$ ratios in the Guijiang River catchment are much higher than those in the Xishui River, reflecting obvious differences in the $\mathrm{Sr}$ isotopic compositions of the underlying bedrock in the two catchments. 
Table 1. The sampling information of the Xishui River and Guijiang River.

\begin{tabular}{|c|c|c|c|c|c|c|c|c|c|}
\hline Num. & River basins & Locations & Date & Longitude & Latitude & $\begin{array}{l}\text { Ele. } \\
(\mathrm{m})\end{array}$ & $\begin{array}{l}\text { Temp. } \\
\left({ }^{\circ} \mathrm{C}\right)\end{array}$ & $\mathrm{pH}$ & $\begin{array}{l}\mathrm{EC} \\
\left(\mu \mathrm{cm}^{-1}\right)\end{array}$ \\
\hline \multicolumn{10}{|c|}{ The Xishui River } \\
\hline \multicolumn{10}{|l|}{ Winter } \\
\hline $\mathrm{XS}-01^{\mathrm{a}}$ & Xishui R. & Lanxi & $25 \operatorname{Dec} 2010$ & $115^{\circ} 08^{\prime} 40^{\prime \prime}$ & $30^{\circ} 21^{\prime} 25^{\prime \prime}$ & 15 & 9 & 7.7 & 183 \\
\hline $\mathrm{XS}-02^{\mathrm{a}}$ & Xishui R. & Guankou & 25 Dec 2010 & $115^{\circ} 20^{\prime} 13^{\prime \prime}$ & $30^{\circ} 32^{\prime} 24^{\prime \prime}$ & 43 & 10.3 & 7.9 & 125 \\
\hline$X S-03^{a}$ & Shenjia R. & Bailianhe & 25 Dec 2010 & $115^{\circ} 25^{\prime} 29^{\prime \prime}$ & $30^{\circ} 37^{\prime} 11^{\prime \prime}$ & 60 & 9.1 & 8 & 183 \\
\hline XS-0 $04^{\mathrm{a}}$ & Xishui R. & Bailianhe & $25 \operatorname{Dec} 2010$ & $115^{\circ} 26^{\prime} 20^{\prime \prime}$ & $30^{\circ} 35^{\prime} 42^{\prime \prime}$ & 61 & 10.7 & 7.8 & 122 \\
\hline$X S-05^{a}$ & Xihe R. & Yinshan & $25 \operatorname{Dec} 2010$ & $115^{\circ} 38^{\prime} 31^{\prime \prime}$ & $30^{\circ} 43^{\prime} 38^{\prime \prime}$ & 101 & 8.2 & 7.9 & 109 \\
\hline$X S-06^{a}$ & Donghe R. & Yangliuwang & 26 Dec 2010 & $115^{\circ} 44^{\prime} 52^{\prime \prime}$ & $30^{\circ} 47^{\prime} 13^{\prime \prime}$ & 131 & 9.2 & 7.8 & 155 \\
\hline $\mathrm{XS}-07^{\mathrm{a}}$ & Xihe R. & Jinjiapu & 26 Dec 2010 & $115^{\circ} 37^{\prime} 53^{\prime \prime}$ & $30^{\circ} 52^{\prime} 05^{\prime \prime}$ & 126 & 6.2 & 7.86 & 93.1 \\
\hline XS-08 ${ }^{a}$ & Xihe R. & Shitouzui & $26 \operatorname{Dec} 2010$ & $115^{\circ} 47^{\prime} 10^{\prime \prime}$ & $31^{\circ} 01^{\prime} 05^{\prime \prime}$ & 198 & 4.5 & 7.8 & 99.1 \\
\hline \multirow[t]{2}{*}{$\mathrm{XS}-09^{\mathrm{a}}$} & Xihe R. & Wujiashan & 26 Dec 2010 & $115^{\circ} 49^{\prime} 35^{\prime \prime}$ & $31^{\circ} 04^{\prime} 28^{\prime \prime}$ & 277 & & & \\
\hline & Snow & Lanxi & 25 Dec 2010 & $115^{\circ} 08^{\prime} 40^{\prime \prime}$ & $31^{\circ} 04^{\prime} 28^{\prime \prime}$ & 22 & & & \\
\hline \multicolumn{10}{|l|}{ Summer } \\
\hline XS-01 & Xishui R. & Lanxi & 9 Jul 2011 & $115^{\circ} 08^{\prime} 40^{\prime \prime}$ & $30^{\circ} 21^{\prime} 25^{\prime \prime}$ & 15 & 28 & & 126 \\
\hline XS-02 & Xishui R. & Guankou & 9 Jul 2011 & $115^{\circ} 20^{\prime} 13^{\prime \prime}$ & $30^{\circ} 32^{\prime} 24^{\prime \prime}$ & 43 & 23 & & 142 \\
\hline XS-03 & Shenjia R. & Bailianhe & 9 Jul 2011 & $115^{\circ} 25^{\prime} 29^{\prime \prime}$ & $30^{\circ} 37^{\prime} 11^{\prime \prime}$ & 60 & 21 & & 131 \\
\hline XS-04 & Xishui R. & Bailianhe & 9 Jul 2011 & $115^{\circ} 26^{\prime} 20^{\prime \prime}$ & $30^{\circ} 35^{\prime} 42^{\prime \prime}$ & 61 & 22 & & 140 \\
\hline XS-05 & Xihe R. & Yinshan & 9 Jul 2011 & $115^{\circ} 38^{\prime} 31^{\prime \prime}$ & $30^{\circ} 43^{\prime} 38^{\prime \prime}$ & 101 & 28 & & 140 \\
\hline XS-06 & Donghe R. & Yangliuwang & 9 Jul 2011 & $115^{\circ} 44^{\prime} 52^{\prime \prime}$ & $30^{\circ} 47^{\prime} 13^{\prime \prime}$ & 131 & 24 & & 160 \\
\hline XS-07 & Xihe R. & Jinjiapu & 9 Jul 2011 & $115^{\circ} 37^{\prime} 53^{\prime \prime}$ & $30^{\circ} 52^{\prime} 05^{\prime \prime}$ & 126 & 22 & & 133 \\
\hline XS-08 & Xihe R. & Shitouzui & 9 Jul 2011 & $115^{\circ} 47^{\prime} 10^{\prime \prime}$ & $31^{\circ} 01^{\prime} 05^{\prime \prime}$ & 198 & 20 & & 109 \\
\hline \multicolumn{10}{|c|}{ The Guijiang River } \\
\hline$G J-01^{a}$ & Lin $\mathrm{R}$. & Rongjiang & 26 Jul 2010 & $110^{\circ} 28^{\prime} 36^{\prime \prime}$ & $25^{\circ} 33^{\prime} 48^{\prime \prime}$ & 184 & 28 & 7.99 & 236 \\
\hline $\mathrm{GJ}-02^{\mathrm{a}}$ & Darong R. & Rongjiang & 26 Jul 2010 & $110^{\circ} 28^{\prime} 18^{\prime \prime}$ & $25^{\circ} 33^{\prime} 52^{\prime \prime}$ & 186 & 27.6 & 7.37 & 71 \\
\hline GJ- $03^{\mathrm{a}}$ & Li R. & Rongjiang & 26 Jul 2010 & $110^{\circ} 27^{\prime} 26^{\prime \prime}$ & $25^{\circ} 33^{\prime} 14^{\prime \prime}$ & 179 & 27.3 & 7.39 & 74 \\
\hline GJ-04 & Gantang R. & Tanxia & 27 Jul 2010 & $110^{\circ} 17^{\prime} 28^{\prime \prime}$ & $25^{\circ} 27^{\prime} 25^{\prime \prime}$ & 168 & 25 & 7.58 & 152 \\
\hline GJ-05 & Li R. & Guilin & 27 Jul 2010 & $110^{\circ} 19^{\prime} 38^{\prime \prime}$ & $25^{\circ} 21^{\prime} 19^{\prime \prime}$ & 154 & 28.2 & 7.62 & 170 \\
\hline GJ-06 & Taohua R. & Guilin & 27 Jul 2010 & $110^{\circ} 17^{\prime} 02^{\prime \prime}$ & $25^{\circ} 16^{\prime} 32^{\prime \prime}$ & 153 & 28.5 & 7.27 & 229 \\
\hline GJ-07 & Li R. & Guilin & 27 Jul 2010 & $110^{\circ} 18^{\prime} 58^{\prime \prime}$ & $25^{\circ} 13^{\prime} 53^{\prime \prime}$ & 148 & 28.1 & 7.3 & 172 \\
\hline GJ-08 & Li R. & Zhemu & 27 Jul 2010 & $110^{\circ} 20^{\prime} 58^{\prime \prime}$ & $25^{\circ} 12^{\prime} 13^{\prime \prime}$ & 146 & 28.7 & 7.31 & 166 \\
\hline GJ-09a & Liangfeng R. & Zhemu & 27 Jul 2010 & $110^{\circ} 21^{\prime} 04^{\prime \prime}$ & $25^{\circ} 11^{\prime} 59^{\prime \prime}$ & 147 & 29.8 & 7.41 & 316 \\
\hline GJ $-10^{\mathrm{a}}$ & Li R. & Majiafang & 27 Jul 2010 & $110^{\circ} 23^{\prime} 13^{\prime \prime}$ & $25^{\circ} 10^{\prime} 44^{\prime \prime}$ & 141 & 29.3 & 7.41 & 177 \\
\hline GJ-11 $1^{\mathrm{a}}$ & Chaotian R. & Daxu & 27 Jul 2010 & $110^{\circ} 25^{\prime} 45^{\prime \prime}$ & $25^{\circ} 10^{\prime} 38^{\prime \prime}$ & 136 & 30.6 & 8.56 & 207 \\
\hline GJ-12 & Li R. & Guanyan & 27 Jul 2010 & $110^{\circ} 26^{\prime} 52^{\prime \prime}$ & $25^{\circ} 03^{\prime} 16^{\prime \prime}$ & 128 & 30.5 & 8.38 & 182 \\
\hline GJ-13 & Underground R. & Guanyan & 27 Jul 2010 & $110^{\circ} 27^{\prime} 25^{\prime \prime}$ & $25^{\circ} 02^{\prime} 57^{\prime \prime}$ & 129 & 22.3 & 8.13 & 258 \\
\hline GJ-14 & Li R. & Guanyan & 27 Jul 2010 & $110^{\circ} 27^{\prime} 14^{\prime \prime}$ & $25^{\circ} 02^{\prime} 38^{\prime \prime}$ & 128 & 30.5 & 8.31 & 182 \\
\hline GJ $-15^{b}$ & Li R. & Xinping & 27 Jul 2010 & $110^{\circ} 31^{\prime} 07^{\prime \prime}$ & $24^{\circ} 55^{\prime} 20^{\prime \prime}$ & 118 & 29.8 & 7.73 & 185 \\
\hline GJ-16 & Longjin R. & Xinping & 28 Jul 2010 & $110^{\circ} 31^{\prime} 35^{\prime \prime}$ & $24^{\circ} 55^{\prime} 04^{\prime \prime}$ & 119 & 28.6 & 7.77 & 228 \\
\hline GJ-17 & Li R. & Xinping & $28 \mathrm{Jul} 2010$ & $110^{\circ} 31^{\prime} 05^{\prime \prime}$ & $24^{\circ} 54^{\prime} 45^{\prime \prime}$ & 118 & 29.8 & 7.78 & 186 \\
\hline GJ-18 & Li R. & Yangshuo & 28 Jul 2010 & $110^{\circ} 30^{\prime} 00^{\prime \prime}$ & $24^{\circ} 46^{\prime} 10^{\prime \prime}$ & 112 & 30.5 & 8.14 & 189 \\
\hline GJ-19 & Yulong R. & Yangshuo & 28 Jul 2010 & $110^{\circ} 30^{\prime} 40^{\prime \prime}$ & $24^{\circ} 46^{\prime} 05^{\prime \prime}$ & 112 & 31.2 & 8.1 & 238 \\
\hline GJ $-20^{\mathrm{a}}$ & Li R. & Yangshuo & 28 Jul 2010 & $110^{\circ} 31^{\prime} 02^{\prime \prime}$ & $24^{\circ} 46^{\prime} 42^{\prime \prime}$ & 112 & 30.6 & 8.2 & 193 \\
\hline GJ-21 & Li R. & Pingle & 28 Jul 2010 & $110^{\circ} 36^{\prime} 44^{\prime \prime}$ & $24^{\circ} 38^{\prime} 28^{\prime \prime}$ & 104 & 30.5 & 7.98 & 191 \\
\hline $\mathrm{GJ}-22^{\mathrm{a}}$ & Lipu R. & Pingle & 28 Jul 2010 & $110^{\circ} 36^{\prime} 43^{\prime \prime}$ & $24^{\circ} 37^{\prime} 57^{\prime \prime}$ & 103 & 30.2 & 7.72 & 159 \\
\hline GJ $-23^{\mathrm{a}}$ & Li R. & Pingle & $28 \mathrm{Jul} 2010$ & $110^{\circ} 37^{\prime} 40^{\prime \prime}$ & $24^{\circ} 37^{\prime} 48^{\prime \prime}$ & 103.5 & 30.3 & 7.96 & 175 \\
\hline $\mathrm{GJ}-24^{\mathrm{a}}$ & Gongcheng R. & Pingle & 28 Jul 2010 & $110^{\circ} 38^{\prime} 15^{\prime \prime}$ & $24^{\circ} 37^{\prime} 58^{\prime \prime}$ & 104 & 30.4 & 8.01 & 201 \\
\hline GJ $-25^{\mathrm{a}}$ & Gui R. & Pingle & 28 Jul 2010 & $110^{\circ} 40^{\prime} 10^{\prime \prime}$ & $24^{\circ} 37^{\prime} 00^{\prime \prime}$ & 102 & 30.1 & 7.93 & 192 \\
\hline GJ-26 & Gui R. & Guihua & 29 Jul 2010 & $110^{\circ} 46^{\prime} 06^{\prime \prime}$ & $24^{\circ} 15^{\prime} 31^{\prime \prime}$ & 76 & 30.4 & 7.71 & 190 \\
\hline GJ-27 & Guihua R. & Guihua & 29 Jul 2010 & $110^{\circ} 45^{\prime} 46^{\prime \prime}$ & $24^{\circ} 15^{\prime} 06^{\prime \prime}$ & 76 & 30.7 & 8.12 & 164 \\
\hline GJ-28 & Gui R. & Guihua & 29 Jul 2010 & $110^{\circ} 47^{\prime} 44^{\prime \prime}$ & $24^{\circ} 14^{\prime} 46^{\prime \prime}$ & 75 & 30.3 & 7.78 & 184 \\
\hline GJ-29 & Gui R. & Zhaoping & 29 Jul 2010 & $110^{\circ} 50^{\prime} 20^{\prime \prime}$ & $24^{\circ} 11^{\prime} 47^{\prime \prime}$ & 55 & 30.3 & 7.81 & 176 \\
\hline GJ $-30^{\mathrm{a}}$ & Siqin R. & Zhaoping & 29 Jul 2010 & $110^{\circ} 50^{\prime} 34^{\prime \prime}$ & $24^{\circ} 11^{\prime} 30^{\prime \prime}$ & 55 & 30 & 8.01 & 142 \\
\hline
\end{tabular}


Table 1. Continued.

\begin{tabular}{|c|c|c|c|c|c|c|c|c|c|}
\hline Num. & River basins & Locations & Date & Longitude & Latitude & $\begin{array}{l}\text { Ele. } \\
(\mathrm{m})\end{array}$ & $\begin{array}{r}\text { Temp. } \\
\left({ }^{\circ} \mathrm{C}\right)\end{array}$ & $\mathrm{pH}$ & $\begin{array}{l}\mathrm{EC} \\
\left(\mu \mathrm{cm}^{-1}\right)\end{array}$ \\
\hline$G J-31^{a}$ & Gui R. & Zhaoping & 29 Jul 2010 & $110^{\circ} 49^{\prime} 57^{\prime \prime}$ & $24^{\circ} 10^{\prime} 48^{\prime \prime}$ & 53 & 28 & 7.76 & 178 \\
\hline GJ-32 & Gui R. & Majiang & 29 Jul 2010 & $111^{\circ} 02^{\prime} 12^{\prime \prime}$ & $23^{\circ} 53^{\prime} 44^{\prime \prime}$ & 33 & 30.3 & 7.73 & 152 \\
\hline$G J-33^{a}$ & Fuqin R. & Majiang & 30 Jul 2010 & $111^{\circ} 02^{\prime} 16^{\prime \prime}$ & $23^{\circ} 52^{\prime} 38^{\prime \prime}$ & 34 & 29.4 & 7.64 & 132 \\
\hline $\mathrm{GJ}-34^{\mathrm{a}}$ & Gui R. & Majiang & 30 Jul 2010 & $111^{\circ} 01^{\prime} 29^{\prime \prime}$ & $23^{\circ} 52^{\prime} 07^{\prime \prime}$ & 32.5 & 30.4 & 7.8 & 152 \\
\hline GJ-35 & Gui R. & Changfa & $30 \mathrm{Jul} 2010$ & $111^{\circ} 05^{\prime} 58^{\prime \prime}$ & $23^{\circ} 43^{\prime} 01^{\prime \prime}$ & 23.5 & 30.3 & 7.76 & 145 \\
\hline GJ-36 & Longjiang R. & Changfa & 30 Jul 2010 & $111^{\circ} 06^{\prime} 09^{\prime \prime}$ & $23^{\circ} 41^{\prime} 51^{\prime \prime}$ & 23 & 26.9 & 7.11 & 36 \\
\hline GJ-37 & Gui R. & Changfa & $30 \mathrm{Jul} 2010$ & $110^{\circ} 07^{\prime} 47^{\prime \prime}$ & $23^{\circ} 38^{\prime} 35^{\prime \prime}$ & 21 & 30.1 & 7.8 & 143 \\
\hline GJ-38 & Gui R. & Hekou & 30 Jul 2010 & $111^{\circ} 18^{\prime} 17^{\prime \prime}$ & $23^{\circ} 32^{\prime} 29^{\prime \prime}$ & 11 & 29.8 & 7.69 & 138 \\
\hline GJ-39 & Siliang R. & Hekou & 31 Jul 2010 & $111^{\circ} 18^{\prime} 32^{\prime \prime}$ & $23^{\circ} 32^{\prime} 04^{\prime \prime}$ & 11 & 28.5 & 6.78 & 89 \\
\hline \multirow[t]{2}{*}{ GJ-40 } & Gui R. & Hekou & 31 Jul 2010 & $111^{\circ} 18^{\prime} 37^{\prime \prime}$ & $23^{\circ} 31^{\prime} 30^{\prime \prime}$ & 10 & 30.5 & 7.67 & 138 \\
\hline & Rain & Zhaoping & 29 Jul 2010 & $110^{\circ} 49^{\prime} 57^{\prime \prime}$ & $24^{\circ} 10^{\prime} 48^{\prime \prime}$ & 53 & & & \\
\hline
\end{tabular}

${ }^{a}$ Riverbed sediments were also collected. ${ }^{\mathrm{b}}$ Riverbed sediments and soils were also collected.
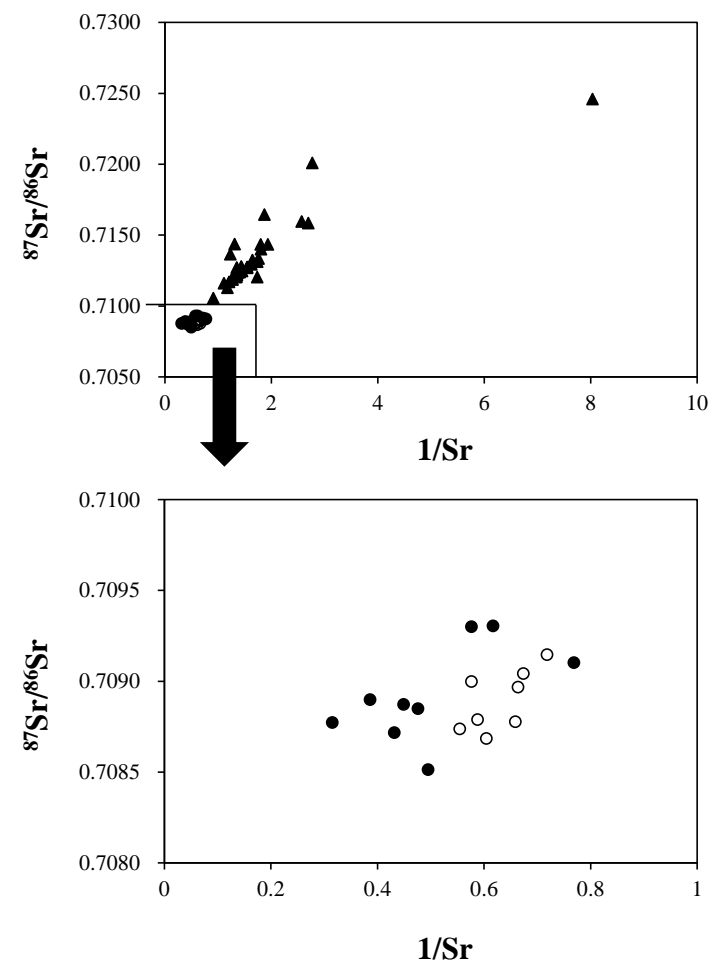

Fig. 5. Plot of the ${ }^{87} \mathrm{Sr} /{ }^{86} \mathrm{Sr}$ ratios vs. $1 / \mathrm{Sr}$ in the Xishui and Guijiang Rivers. The filled triangles represent the samples in the Guijiang River, and the filled and open circles represent the samples in the Xishui River in the winter and summer, respectively.

\section{Discussion}

\subsection{Sr isotope characteristics and controlling factors in the Xishui River}

Silicate rocks in the Xishui River catchment account for approximately $95 \%$ of the drainage area. Among them, gneisses are dominant and granites are mainly distributed in
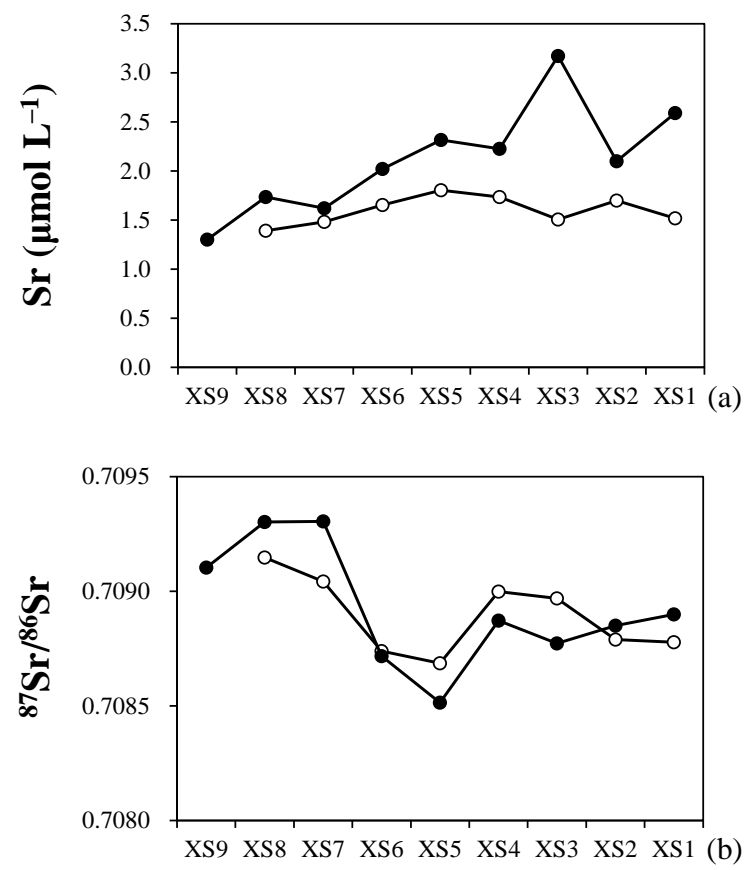

Fig. 6. Temporal and spatial variations of the Sr concentrations (a) and the ${ }^{87} \mathrm{Sr} /{ }^{86} \mathrm{Sr}$ ratios (b) in the Xishui River. The filled and open circles represent the samples in the winter and summer, respectively.

the source area and in the Bailianhe Reservoir. Moreover, basic and ultrabasic rocks are exposed in a scattered manner. However, as a silicate watershed, the Xishui River has low ${ }^{87} \mathrm{Sr} /{ }^{86} \mathrm{Sr}$ ratios $(<0.71)$. The ${ }^{87} \mathrm{Sr} /{ }^{86} \mathrm{Sr}$ ratios of samples in the summer decrease gradually going downwards from the source area and reach the lowest value at Yinshan County (Fig. 6). After flowing across granites, which are widely distributed across the Bailianhe Reservoir, the ${ }^{87} \mathrm{Sr} /{ }^{86} \mathrm{Sr}$ ratios increase and then gradually decrease from XS3 to XS1. The varying trend of ${ }^{87} \mathrm{Sr} /{ }^{86} \mathrm{Sr}$ ratios in the winter is very similar to that of the summer with the exception of XS3-XS1, 
Table 2. The Sr isotopic compositions and parts of major ion concentrations in the Xishui River and Guijiang River.

\begin{tabular}{|c|c|c|c|c|c|c|c|c|c|c|c|c|c|c|}
\hline Num. & $\begin{array}{l}\text { River } \\
\text { basins }\end{array}$ & $\mathrm{Ca}$ & $\mathrm{Mg}$ & $\mathrm{K}$ & $\mathrm{Na}$ & & $\begin{array}{l}\mathrm{HCO}_{3} \\
1 \mathrm{~L}^{-1}\end{array}$ & $\mathrm{SO}_{4}$ & $\mathrm{NO}_{3}$ & $\mathrm{Si}$ & $\mathrm{Sr}$ & ${ }^{87} \mathrm{Sr} /{ }^{86} \mathrm{Sr}$ & $\begin{array}{l}2 \sigma \\
\%\end{array}$ & NICB \\
\hline \multicolumn{15}{|c|}{ The Xishui River ${ }^{\mathrm{a}}$} \\
\hline \multicolumn{15}{|l|}{ Winter } \\
\hline XS-01 & Xishui R. & 427 & 201 & 79.2 & 384 & 149 & 1320 & 119 & 112 & 220 & 2.59 & 0.708900 & 3 & 0.3 \\
\hline XS-02 & Xishui R. & 274 & 125 & 41.1 & 261 & 92.8 & 872 & 100 & 46.3 & 197 & 2.10 & 0.708850 & 4 & -2.9 \\
\hline XS-03 & Shengjiahe R. & 491 & 233 & 49.0 & 361 & 113 & 1537 & 122 & 28.9 & 278 & 3.17 & 0.708773 & 6 & -1.0 \\
\hline XS-04 & Xishui R. & 289 & 131 & 44.8 & 285 & 86.9 & 918 & 95.4 & 47.3 & 220 & 2.23 & 0.708873 & 3 & -1.1 \\
\hline XS-05 & Xihe R. & 252 & 120 & 35.1 & 268 & 87.9 & 684 & 119 & 35.7 & 206 & 2.02 & 0.708515 & 4 & 1.8 \\
\hline XS-06 & Donghe R. & 345 & 153 & 39.4 & 425 & 126 & 930 & 155 & 34.2 & 307 & 2.32 & 0.708718 & 5 & 3.3 \\
\hline XS-07 & Xihe R. & 211 & 93.4 & 29.8 & 230 & 50.2 & 621 & 114 & 51.1 & 164 & 1.62 & 0.709305 & 3 & -1.7 \\
\hline XS-08 & Xihe R. & 231 & 109 & 28.5 & 218 & 57.0 & 690 & 118 & 39.8 & 199 & 1.73 & 0.709302 & 2 & -2.9 \\
\hline \multirow[t]{2}{*}{ XS-09 } & Xihe R. & 130 & 54.0 & 18.6 & 149 & 31.8 & 394 & 70.9 & 16.7 & 224 & 1.30 & 0.709104 & 2 & -2.9 \\
\hline & Snow & 66.4 & 13.1 & 21.1 & 38.4 & 38.9 & 105 & 32.4 & 93.3 & 4.04 & 0.308 & 0.709495 & 4 & -38.4 \\
\hline \multicolumn{15}{|l|}{ Summer } \\
\hline XS-01 & Xishui R. & 268 & 151 & 46.2 & 291 & 168 & 730 & 133 & 66.8 & 130 & 1.52 & 0.708778 & 3 & -2.1 \\
\hline XS-02 & Xishui R. & 324 & 151 & 47.0 & 366 & 155 & 905 & 127 & 51.6 & 200 & 1.70 & 0.708790 & 5 & 1.8 \\
\hline XS-03 & Shengjiahe R. & 287 & 138 & 45.4 & 324 & 140 & 801 & 116 & 51.1 & 132 & 1.51 & 0.708969 & 7 & 2.0 \\
\hline XS-04 & Xishui R. & 341 & 146 & 50.4 & 351 & 135 & 883 & 124 & 53.7 & 179 & 1.14 & 0.708999 & 3 & 2.2 \\
\hline XS-05 & Xihe R. & 313 & 139 & 43.5 & 361 & 153 & 847 & 162 & 55.6 & 194 & 0.468 & 0.708686 & 5 & 0.1 \\
\hline XS-06 & Donghe R. & 371 & 150 & 44.6 & 432 & 170 & 1011 & 146 & 51.6 & 281 & 0.890 & 0.708739 & 4 & -1.8 \\
\hline XS-07 & Xihe R. & 258 & 128 & 37.7 & 396 & 130 & 765 & 153 & 38.7 & 154 & 1.48 & 0.709043 & 5 & -1.3 \\
\hline XS-08 & Xihe R. & 250 & 120 & 33.5 & 263 & 109 & 603 & 150 & 32.3 & 153 & 1.39 & 0.709148 & 8 & -0.3 \\
\hline \multicolumn{15}{|c|}{ The Guijiang River ${ }^{b}$} \\
\hline GJ-01 & Lin $R$. & & & & & & & & & & 0.851 & 0.711293 & 6 & \\
\hline GJ-02 & Darong R. & 286 & 62.6 & 20 & 60.9 & 48.8 & 370 & 88.5 & 131 & & 0.371 & 0.715849 & 6 & \\
\hline GJ-03 & Li R. & & & & & & & & & & 0.389 & 0.715973 & 3 & \\
\hline GJ-04 & Gantang R. & 335 & 21.0 & 8.97 & 21.7 & 27.9 & 630 & 47.3 & 43.4 & & 0.576 & 0.712037 & 4 & \\
\hline GJ-05 & Li R. & 797 & 73.3 & 31.8 & 141 & 134 & 1600 & 117 & 129 & & 1.098 & 0.710558 & 5 & \\
\hline GJ-06 & Taohua R. & & & & & & & & & & 0.743 & 0.712451 & 5 & \\
\hline GJ-07 & Li R. & & & & & & & & & & 0.786 & 0.711876 & 6 & \\
\hline GJ-08 & Li R. & & & & & & & & & & 0.737 & 0.712369 & 12 & \\
\hline GJ-09 & Liangfeng R. & & & & & & & & & & 0.899 & 0.711612 & 4 & \\
\hline GJ-10 & Li R. & & & & & & & & & & 0.741 & 0.712728 & 50 & \\
\hline GJ-11 & Chaotian R. & 967 & 128 & 17.7 & 34.4 & 58.4 & 2099 & 79.8 & 93.1 & & 0.811 & 0.713659 & 3 & \\
\hline GJ-12 & Li R. & & & & & & & & & & 0.754 & 0.712393 & 35 & \\
\hline GJ-13 & Underground R. & & & & & & & & & & 0.743 & 0.712359 & 4 & \\
\hline GJ-14 & Li R. & & & & & & & & & & 0.749 & 0.712077 & 3 & \\
\hline GJ-15 & Li R. & & & & & & & & & & 0.745 & 0.712084 & 4 & \\
\hline GJ-16 & Longjin R. & & & & & & & & & & 0.764 & 0.714377 & 3 & \\
\hline GJ-17 & Li R. & & & & & & & & & & 0.738 & 0.712204 & 4 & \\
\hline GJ-18 & Li R. & & & & & & & & & & 0.750 & 0.712039 & 4 & \\
\hline GJ-19 & Yulong R. & 1327 & 346 & 20.3 & 57.8 & 97.6 & 3130 & 135 & 133 & & 0.694 & 0.712706 & 5 & \\
\hline GJ-20 & Li R. & 893 & 95.9 & 31.3 & 111 & 107 & 1754 & 130 & 73.7 & & 0.743 & 0.712200 & 8 & \\
\hline GJ-21 & Li R. & & & & & & & & & & 0.746 & 0.712078 & 7 & \\
\hline GJ-22 & Lipu R. & 700 & 189 & 50.8 & 127 & 121 & 1250 & 249 & 67.1 & & 0.829 & 0.711712 & 6 & \\
\hline GJ-23 & LiR. & & & & & & & & & & 0.792 & 0.711911 & 4 & \\
\hline GJ-24 & Gongcheng R. & 705 & 174 & 25.9 & 53.5 & 63.8 & 1320 & 96.1 & 107 & & 0.702 & 0.712568 & 6 & \\
\hline GJ-25 & Gui R. & & & & & & & & & & 0.727 & 0.712372 & 3 & \\
\hline GJ-26 & Gui R. & & & & & & & & & & 0.711 & 0.712402 & 4 & \\
\hline GJ-27 & Guihua R. & & & & & & & & & & 0.615 & 0.712943 & 6 & \\
\hline GJ-28 & Gui R. & & & & & & & & & & 0.689 & 0.712483 & 3 & \\
\hline GJ-29 & Gui R. & & & & & & & & & & 0.647 & 0.712709 & 6 & \\
\hline GJ-30 & Siqin R. & 743 & 163 & 46.7 & 118 & 114 & 1688 & 114 & 62.4 & & 0.607 & 0.713242 & 3 & \\
\hline GJ-31 & Gui R. & 816 & 134 & 35.1 & 99.1 & 98.4 & 1655 & 137 & 58.7 & & 0.646 & 0.712750 & 7 & \\
\hline GJ-32 & Gui R. & & & & & & & & & & 0.567 & 0.713365 & 4 & \\
\hline GJ-33 & Fuqin R. & 478 & 145 & 45.6 & 82.2 & 88.6 & 1100 & 90.6 & 60.2 & & 0.697 & 0.712798 & 4 & \\
\hline GJ-34 & Gui R. & & & & & & & & & & 0.576 & 0.713128 & 5 & \\
\hline GJ-35 & Gui R. & 690 & 138 & 31.3 & 87.4 & 84.9 & 1450 & 116 & 51.0 & & 0.555 & 0.714357 & 8 & \\
\hline GJ-36 & Longjiang R. & 78.5 & 74.1 & 33.8 & 106 & 47.4 & 270 & 48.6 & 56.5 & & 0.124 & 0.724605 & 5 & \\
\hline GJ-37 & Gui R. & & & & & & & & & & 0.554 & 0.714018 & 5 & \\
\hline GJ-38 & Gui R. & & & & & & & & & & 0.516 & 0.714343 & 3 & \\
\hline GJ-39 & Siliang R. & & & & & & & & & & 0.361 & 0.720095 & 3 & \\
\hline GJ-40 & Gui R. & & & & & & & & & & 0.534 & 0.716453 & 4 & \\
\hline Rain & Rain & & & & & & & & & & 0.110 & 0.710416 & 3 & \\
\hline
\end{tabular}

${ }^{a}$ Major ion concentrations in the Xishui River were from a companion paper (Wu et al., 2013). ${ }^{b}$ Major ion concentrations in the Guijiang River were cited from H. B. Zhang et al. (2012). Compared with our samples, they were collected at a different time (April 2012), and therefore can only be a reference. 
Table 3. Sr isotopic compositions of the Xishui River and Guijiang River riverbed sediments and soils.

\begin{tabular}{lccc}
\hline Num. & $\mathrm{Sr}\left(\mu \mathrm{g} \mathrm{g}^{-1}\right)$ & ${ }^{87} \mathrm{Sr} /{ }^{86} \mathrm{Sr}$ & $2 \sigma$ \\
\hline \multicolumn{4}{c}{ The Xishui River sediments } \\
\hline XS-01 & 249 & 0.710139 & 2 \\
XS-02 & 354 & 0.709188 & 3 \\
XS-03 & 255 & 0.712616 & 3 \\
XS-04 & 232 & 0.711184 & 4 \\
XS-05 & 396 & 0.707058 & 3 \\
XS-06 & 423 & 0.708789 & 4 \\
XS-07 & 362 & 0.709222 & 3 \\
XS-08 & 378 & 0.709050 & 4 \\
XS-09 & 448 & 0.708784 & 3 \\
\hline \multicolumn{4}{c}{ The Guijiang River sediments } \\
\hline GJ-01 & 41.6 & 0.743134 & 3 \\
GJ-02 & 43.9 & 0.757536 & 4 \\
GJ-03 & 56.6 & 0.738083 & 4 \\
GJ-09 & 61.6 & 0.739180 & 3 \\
GJ-10 & 54.6 & 0.742779 & 2 \\
GJ-11 & 58.1 & 0.742161 & 4 \\
GJ-15 & 49.1 & 0.744003 & 3 \\
GJ-19 & 63.6 & 0.737776 & 3 \\
GJ-20 & 47.8 & 0.758427 & 3 \\
GJ-22 & 87.0 & 0.737306 & 4 \\
GJ-23 & 77.3 & 0.737048 & 4 \\
GJ-24 & 85.1 & 0.735172 & 3 \\
GJ-25 & 93.8 & 0.735274 & 3 \\
GJ-30 & 55.1 & 0.748129 & 4 \\
GJ-31 & 59.6 & 0.740296 & 5 \\
GJ-33 & 38.3 & 0.775952 & 4 \\
GJ-34 & 52.2 & 0.761448 & 4 \\
GJ-40 & 58.3 & 0.750115 & 4 \\
\hline \multicolumn{4}{c}{ The Guijiang River soils } \\
GJ-15 & 55.4 & 0.744095 & 5 \\
\hline
\end{tabular}

which have a slightly increasing trend. Variations in the ${ }^{87} \mathrm{Sr} /{ }^{86} \mathrm{Sr}$ ratios may be attributed to differences in the underlying bedrock. In the Xishui River catchment, the ${ }^{87} \mathrm{Sr} /{ }^{86} \mathrm{Sr}$ ratios of Cretaceous granites are commonly higher than those of metamorphic rocks (Zheng et al., 2000; Ge et al., 2001a, b; Chen et al., 2002). The Sr concentrations in the Xishui River are relatively high and the lower reaches have higher values than do the headwaters. The samples XS8-XS4 in the summer and winter have similar trends of increasing $\mathrm{Sr}$ concentrations. However, the varying trend of samples XS3XS1 between the summer and winter is remarkably different, as reflected in the very high $\mathrm{Sr}$ concentrations of samples XS3 and XS1 in the winter. Moreover, the concentrations of the major ions $\mathrm{Ca}^{2+}, \mathrm{Mg}^{2+}$, and $\mathrm{HCO}_{3}^{-}$of samples XS3 and XS1 were also rather high. This result may reflect hydrological control at the basin scale. The river water is
Table 4. Sr isotopic compositions of underlying bedrocks in the Xishui River and Guijiang River catchments.

\begin{tabular}{lcl}
\hline Lithologies & ${ }^{87} \mathrm{Sr} /{ }^{86} \mathrm{Sr}$ & Data Source \\
\hline & The Xishui River catchment \\
\hline Granitoids & $0.708229-0.716990$ & Ge et al. (2001a) \\
Granitoids & $0.707934-0.713695$ & Chen et al. (2002) \\
Granitoids & $0.707040-0.712890$ & Zhang et al. (2002) \\
Gray Gneisses & $0.707482-0.719772$ & Zheng et al. (2000) \\
Pyroxenite/gabbro & $0.706839-0.708556$ & Jahn et al. (1999) \\
Mafic-ultramafic Rocks & $0.706071-0.703955$ & Li et al. (1998) \\
Eclogites & $0.705388-0.710926$ & Liu et al. (2000) \\
Complex & $0.707109-0.707611$ & Wawrzenitz et al. (2006) \\
Mafic igneous & $0.707791-0.709900$ & Wang et al. (2005) \\
\hline & The Guijiang River catchment \\
\hline Granitoids & $0.72261-0.99180$ & Zhu et al. (1989) \\
Granitoids & $0.733000-1.025912$ & Gu et al. (2006) \\
Granitoids & $0.738-1.003$ & Xu and Zhang (1993) \\
Granitoids & $0.77007-0.89397$ & Xu et al. (1994) \\
Granitoids & $0.720065-0.787221$ & F. F. Zhang et al. (2012) \\
Carbonate rocks ${ }^{\mathrm{a}}$ & $0.70589-0.70882$ & Huang (1997) \\
Carbonate rocks ${ }^{\mathrm{a}}$ & $0.708223-0.708907$ & Zeng et al. (2007) \\
Dolomitic limestone ${ }^{\mathrm{b}}$ & 0.70775 & Liu et al. (2011) \\
Soil ${ }^{\mathrm{b}}$ & $0.71049-0.72266$ & Zhu et al. (2011) \\
Soil $^{\mathrm{b}}$ & $0.727317-0.727417$ & \\
\hline
\end{tabular}

${ }^{a}$ Paleozoic carbonate rocks in the Yangtze Platform.

${ }^{\mathrm{b}}$ Karst area in the neighboring Guizhou Province.

mainly provided by surface flow during the monsoon period, whereas the proportion of water contributed by the aquifer is higher during the dry period. The 1:200000 geological map (Bureau of Geology and Mineral Resources of Hubei Province, data not published) shows that Archean marbles are distributed in a stratified manner in the tributary Shenjiahe River and in the Xishui mainstream from Lanxi Town to Xishui County. Therefore, the high concentrations of $\mathrm{Ca}^{2+}$, $\mathrm{Mg}^{2+}, \mathrm{HCO}_{3}^{-}$, and $\mathrm{Sr}$ in the two samples may be caused by increasing carbonate dissolution in the shallow aquifer, whereas the river water is typically derived from the upper reaches in the summer (Wu et al., 2013).

Seasonally, the $\mathrm{Sr}$ concentrations in the summer are obviously lower than those in winter, reflecting a dilution effect resulting from increasing discharge. Variations in the ${ }^{87} \mathrm{Sr} /{ }^{86} \mathrm{Sr}$ ratios are slight and do not exhibit notable regularity. As the Xishui River only has a length of $157 \mathrm{~km}$, the climate effect (temperature, rainfall, evaporation, etc.) among different samples can be negligible. In field reconnaissance, we found that the vegetation type and coverage were similar in the catchment. Studies on the major ions showed that the water residence time in the Xishui River was far shorter than the typical seasonal variation and therefore did not evidently affect the chemical composition of river water, but that the hydrological properties might affect the seasonal differences (e.g., the influence of an aquifer) (Wu et al., 2013). Due to the lack of data on the $\mathrm{Sr}$ isotope in the aquifer, the influence of the aquifer on the seasonal variations of the ${ }^{87} \mathrm{Sr} /{ }^{86} \mathrm{Sr}$ ratios in the Xishui River is unknown. In Table 4, we compile some 
Table 5. Sr isotopic compositions of fertilizer, wastewater, groundwater, and plant in different land use patterns in karst area.

\begin{tabular}{llll}
\hline & $\mathrm{Sr}\left(\mu \mathrm{g} \mathrm{g}^{-1}\right)$ & ${ }^{87} \mathrm{Sr} /{ }^{86} \mathrm{Sr}$ & Data sources \\
\hline Irrigation water & 0.039 & 0.71234 & \\
Basal fertilizer & $0.017-0.057$ & $0.70827-0.70967$ & Hosono et al. (2007) \\
Supplemental fertilizer & $0.044-0.084$ & $0.70894-0.71020$ & \\
fertilizer & & 0.7083 & Négrel and Deschamps (1996) \\
Liquid fertilizer* & 0.948 & 0.708078 & Brenot et al. (2008) \\
Solid fertilizer & 202 & 0.703313 & \\
Winter sewage* & $6.28-10.3$ & 0.70804 & Lang et al. (2006) \\
Summer sewage* & $4.91-8.90$ & 0.70800 & \\
Industrial wastewater* & 10.3 & 0.70766 & \\
Domestic wastewater* & $4.57-5.02$ & $0.70762-0.70820$ & Li et al. (2010) \\
Farmland spring* & $0.92-6.62$ & $0.70794-0.70848$ & \\
Residential area spring* & $1.26-4.45$ & $0.70818-0.70835$ & \\
Cultivated land groundwater* & $2.97-5.63$ & $0.70814-0.71097$ & \\
Grassland groundwater* & $1.93-2.42$ & $0.70758-0.70962$ & \multirow{2}{*}{ Jiang et al. (2009) } \\
Construction land groundwater* & $3.71-7.63$ & $0.70994-0.71089$ & \\
Forested land groundwater * & $0.82-2.05$ & $0.70778-0.70942$ & \\
Plants & $4.9-222$ & $0.70856-0.71145$ & Zheng et al. (2008) \\
\hline
\end{tabular}

* The unit of $\mathrm{Sr}$ concentrations is $\mu \mathrm{mol} \mathrm{L}^{-1}$.

Sr isotopic data of bedrocks in the Xishui River catchment. The ${ }^{87} \mathrm{Sr} /{ }^{86} \mathrm{Sr}$ ratios of these silicate rocks are low and relatively homogeneous with a small variation range. Therefore, the ${ }^{87} \mathrm{Sr} /{ }^{86} \mathrm{Sr}$ ratios of the underlying bedrock are certainly important factors controlling the Sr isotope characteristics in the Xishui River.

Moreover, different land use (agricultural, industrial, and residential use) introduces new sources of weatherable $\mathrm{Sr}$ into rivers. Jiang et al. (2009) and Li et al. (2010) investigated the groundwater in cultivated land, grassland, construction land, and forest land, and the industrial and domestic waste in southwestern China and resulted in ${ }^{87} \mathrm{Sr} /{ }^{86} \mathrm{Sr}$ ratios of 0.70762 to 0.71273 . The ${ }^{87} \mathrm{Sr} /{ }^{86} \mathrm{Sr}$ ratios of 24 fertilizers that are commonly used in Spain range from 0.703350 to 0.715216 with an average of 0.70823 (Vitòria et al., 2004). Brenot et al. (2008) measured the ${ }^{87} \mathrm{Sr} /{ }^{86} \mathrm{Sr}$ ratios of liquid and solid fertilizers (0.708078 and 0.703313 , respectively) in their studies on a small catchment in the Paris Basin. The ${ }^{87} \mathrm{Sr} /{ }^{86} \mathrm{Sr}$ ratios of 12 plants range from 0.70856 to 0.71145 in Guizhou Province (Zheng et al., 2008) (Table 5). As the ${ }^{87} \mathrm{Sr} /{ }^{86} \mathrm{Sr}$ ratios of these potential sources are close to those of the Xishui River water, it is difficult to separate their respective contributions. Studies on the major ions have shown that samples in the summer have higher $\mathrm{Na}^{+}, \mathrm{K}^{+}, \mathrm{Cl}^{-}, \mathrm{NO}_{3}^{-}$, and $\mathrm{SO}_{4}^{2-}$ concentrations than those in the winter, reflecting the influence of agricultural activities and acid rain (Wu et al., 2013). In the next section, we will use an inversion model to identify the influence of anthropogenic activities.

\subsection{Source of $\mathrm{Sr}$ in the Xishui River}

The $\mathrm{Sr}$ in river water is mainly from atmospheric input $\left(\mathrm{Sr}_{\mathrm{atm}}\right)$, anthropogenic activities - urban sewage $\left(\mathrm{Sr}_{\text {urb }}\right)$ and agricultural activities $\left(\mathrm{Sr}_{\mathrm{agr}}\right)$ - silicate weathering $\left(\mathrm{Sr}_{\mathrm{sil}}\right)$, carbonate weathering $\left(\mathrm{Sr}_{\mathrm{carb}}\right)$, and evaporite dissolution $\left(\mathrm{Sr}_{\mathrm{ev}}\right)$ (Galy and France-Lanord, 1999). To quantify the relative contributions of the six endmembers, an inversion model is used (Négrel et al., 1993; Gaillardet et al., 1999; Millot et al., 2003; Wu et al., 2005; Chetelat et al., 2008). The set of mass balance equations is as follows (Negrél et al., 1993; Gaillardet et al., 1999):

$$
\begin{aligned}
& \left(\frac{X}{\mathrm{Na}}\right)_{\text {river }}=\sum_{i}\left(\frac{X}{\mathrm{Na}}\right)_{i} \alpha_{i, \mathrm{Na}} \\
& \left(\frac{{ }^{87} \mathrm{Sr}}{{ }^{86} \mathrm{Sr}}\right)_{\text {river }}\left(\frac{\mathrm{Sr}}{\mathrm{Na}}\right)_{\text {river }}=\sum_{i}\left(\frac{{ }^{87} \mathrm{Sr}}{{ }^{86 \mathrm{Sr}}}\right)_{i}\left(\frac{\mathrm{Sr}}{\mathrm{Na}}\right)_{i} \alpha_{i, \mathrm{Na}},
\end{aligned}
$$

where $X$ represents $\mathrm{Ca}, \mathrm{Mg}, \mathrm{K}, \mathrm{Cl}, \mathrm{NO}_{3}$, and $\mathrm{Sr} ; i$ represents the six endmembers; and $\alpha_{i, \mathrm{Na}}$ represents the respective mass fractions of $\mathrm{Na}$ from different sources. The closure equation is as follows:

$\sum_{i} \alpha_{i, \mathrm{Na}}=1$

Although the Xishui River is a silicate watershed, the lithologies in the catchment are complex, and carbonate rocks are distributed in a stratified and lenticular manner in metamorphic rocks. Therefore, the Na-normalized ratios analyzed in the Xishui River are not suitable for representing the silicate endmember. For the Na-normalized ratios of the silicate endmember, the data of some "truly small watersheds draining 
Table 6. The estimated end member values in the Xishui River.

\begin{tabular}{llllllll}
\hline & $\mathrm{Ca} / \mathrm{Na}$ & $\mathrm{Mg} / \mathrm{Na}$ & $\mathrm{K} / \mathrm{Na}$ & $\mathrm{Cl} / \mathrm{Na}$ & $1000 \times \mathrm{Sr} / \mathrm{Na}$ & $\mathrm{NO}_{3} / \mathrm{Na}$ & ${ }^{87} \mathrm{Sr} /{ }^{86} \mathrm{Sr}$ \\
\hline Rain & $2.0-8.0$ & $0.35-0.85$ & $0.3-0.7$ & $1.7-3.9$ & $10-50$ & $0.1-0.9$ & $0.708-0.709$ \\
Urban & $0.6-1$ & $0.1-0.3$ & $0.09-0.23$ & $0.45-0.75$ & $1.5-2.7$ & 0 & $0.7101-0.7102$ \\
Agriculture & 0 & 0 & $1-1.8$ & $4.0-6.0$ & 0 & $3.0-5.0$ & $0.707-0.709$ \\
Evaporite & $0.15-5$ & $0.01-0.5$ & 0 & 1 & $0.5-5.0$ & 0 & $0.708-0.709$ \\
Carbonate & $30-70$ & $10.0-28.0$ & 0 & 0 & $6.0-12.0$ & 0 & $0.708-0.709$ \\
Silicate & $0.2-1$ & $0.15-0.5$ & $0.1-0.3$ & 0 & $2.0-4.0$ & 0 & $0.72-0.73$ \\
\hline
\end{tabular}

Table 7. The contributions of different sources to $\mathrm{Sr}$ in the Xishui River solved by an inversion model.

\begin{tabular}{lllclll}
\hline & Atmospheric & Urban & Agriculture & Evaporite & Carbonate & Silicate \\
\hline \multirow{2}{*}{ Winter } & $(10.9 \pm 2.9)-$ & $(0.1 \pm 0.1)-$ & & $(0.2 \pm 0.01)-$ & $(7.5 \pm 1.9)-$ & $(30.8 \pm 2.5)-$ \\
& $(57.1 \pm 4.2)$ & $(0.5 \pm 0.3)$ & 0 & $(18.7 \pm 2.0)$ & $(25.3 \pm 6.5)$ & $(53.5 \pm 2.9)$ \\
\hline \multirow{2}{*}{ Summer } & $(1.1 \pm 0.4)-$ & $(0.1 \pm 0.05)-$ & & $(7.3 \pm 2.2)-$ & $(13.2 \pm 2.4)-$ & $(41.5 \pm 3.8)-$ \\
& $(22.0 \pm 4.1)$ & $(1.3 \pm 0.4)$ & 0 & $(17.0 \pm 4.1)$ & $(22.3 \pm 4.5)$ & $(65.6 \pm 5.4)$ \\
\hline
\end{tabular}

silicate rocks" (with a drainage area $<10 \mathrm{~km}^{2}$ ) are referenced (Edmond et al., 1994; White and Blum, 1995; Oliva et al., 2003). The ratios are 0.2 to 1.0 for $\mathrm{Ca} / \mathrm{Na}, 0.15$ to 0.5 for $\mathrm{Mg} / \mathrm{Na}, 0.1$ to 0.3 for $\mathrm{K} / \mathrm{Na}, 0.002$ to 0.004 for $\mathrm{Sr} / \mathrm{Na}$, and 0 for $\mathrm{Cl} / \mathrm{Na}$ and $\mathrm{NO}_{3} / \mathrm{Na}$ (Wu et al., 2013; Table 6). For the other five endmembers, the Na-normalized ratios in the Yangtze River from other authors are used (Chetelat et al., 2008 and references therein; Table 6). Starting from an a priori set of end-member compositions, $(X / \mathrm{Na})_{i}$, we iteratively solved for the proportion of those six endmembers in each sample $\left(\alpha_{i, \mathrm{Na}}\right)$ and the endmember compositions themselves, $(X / \mathrm{Na})_{i}$. The global optimization process within the optimization software package identifies the solution that best predicts the measured compositions in the least-squares sense and propagates the errors (Wu et al., 2013). The standard deviation of the a posteriori values from the a priori ones (relative to the a priori errors) is systematically computed and checked for consistency. The results show that the relative standard deviation of the different $\alpha_{i, \mathrm{Na}}$ is $< \pm 5 \%$ for the silicate source and $< \pm 20 \%$ for the evaporite and carbonate sources, whereas for the atmospheric source, it can reach $30 \%$. Due to the low contribution from urban sewage $\left(\alpha_{\text {urban,Na }}<1 \%\right.$ ), the uncertainties can reach up to $100 \%$. The calculated contributions of the different sources of $\mathrm{Sr}$ in the river are listed in Table 7.

As shown in Table 7, the $\mathrm{Sr}$ in the Xishui River results mainly from silicate weathering, atmospheric input, and carbonate weathering, followed by evaporite dissolution. The influence of anthropogenic activities is negligible. Although the distributed area of carbonate rocks is only $<5 \%$ of the drainage area, it has a disproportionately important contribution to the $\mathrm{Sr}$ in the Xishui River (average 19\%), which is consistent with the conclusion of the studies of the major ions (Wu et al., 2013). Previous studies have indicated that trace calcite in small watersheds draining silicate rocks could contribute a large proportion of the major ions in the river (Blum et al., 1998; Jacobson et al., 2002b; Oliva et al., 2004). As the ${ }^{87} \mathrm{Sr} /{ }^{86} \mathrm{Sr}$ ratios of silicate rocks in the Xishui River catchment are very close to those of Paleozoic carbonate rocks in the Yangtze Platform (Table 4), even if the contribution to $\mathrm{Sr}$ from carbonate weathering far exceeds its distributed area, it still has no obvious decreasing influence on the ${ }^{87} \mathrm{Sr} /{ }^{86} \mathrm{Sr}$ ratios of the river water.

\subsection{Sr isotope characteristics and controlling factors in the Guijiang River}

The Guijiang River flows across the karst region intercalated by detrital rocks, sand rocks, mud rocks, and shales in southern China; granites are only exposed in the source area Miaoershan Mountain and in the upper reaches of the tributary Siqin River. The ${ }^{87} \mathrm{Sr} /{ }^{86} \mathrm{Sr}$ ratios range from 0.719558 to 0.724605 , and the $\mathrm{Sr}$ concentrations range from 0.124 to $1.098 \mu \mathrm{mol} \mathrm{L}^{-1}$ in the Guijiang River. There is a strong positive correlation between the ${ }^{87} \mathrm{Sr} /{ }^{86} \mathrm{Sr}$ ratios and $1 / \mathrm{Sr}$ $\left(r^{2}=0.81\right)$, indicating two-component mixing between silicate and carbonate weathering. Studies from other researchers have shown that granites in the Guijiang River catchment are characterized by extremely high ${ }^{87} \mathrm{Sr} /{ }^{86} \mathrm{Sr}$ ratios with an average of approximately 0.8 (Table 4). Moreover, the ${ }^{87} \mathrm{Sr} /{ }^{86} \mathrm{Sr}$ ratios of the silicate fraction of the riverbed sediments and soils in the Guijiang River catchment range from 0.735172 to 0.775952 with an average of 0.745891 , indicating that these silicate components have high $\mathrm{Sr}$ isotope values. The ${ }^{87} \mathrm{Sr} /{ }^{86} \mathrm{Sr}$ ratios of the soils in the karst cave and in the profiles of the neighboring Guizhou Province range from 0.727317 to 0.727417 and 0.71049 to 0.72266 , respectively (Liu et al., 2011; Zhu et al., 2011). As the groundwater in different land use areas, industrial and 
domestic waste, fertilizers, and plants has low ${ }^{87} \mathrm{Sr} /{ }^{86} \mathrm{Sr}$ ratios (almost entirely $<0.71$, Table 5), it is not the source of the high $\mathrm{Sr}$ isotopic compositions of the Guijiang River water. Therefore, those silicate components in the karst area should be the most important endmembers controlling the $\mathrm{Sr}$ isotopic characteristics of the river water. As we lack $\mathrm{Sr}$ isotope data of the carbonate rocks in the Guijiang River catchment, the ${ }^{87} \mathrm{Sr} /{ }^{86} \mathrm{Sr}$ ratios of the Late Paleozoic marine carbonate rocks in the Yangtze Platform and karst area of the neighboring province are used as other endmembers (0.705890 to 0.708907, Huang, 1997; Zeng et al., 2007; Liu et al., 2011, Table 4). These carbonate rocks are very close in age to those in the Guijiang River catchment, and their ${ }^{87} \mathrm{Sr} /{ }^{86} \mathrm{Sr}$ ratios are therefore used in many studies in the karst area in southwestern China (e.g., Han et al., 2010; Han and Liu, 2004; Jiang et al., 2009). Moreover, these carbonate rocks in the stable Yangtze Craton did not undergo intense tectonism, and thus have no influence on the radiogenic $\mathrm{Sr}$ from the local exchange with silicate rocks.

In the following, we discuss only the spatial variations of the ${ }^{87} \mathrm{Sr} /{ }^{86} \mathrm{Sr}$ ratios due to the strong positive correlation between the ${ }^{87} \mathrm{Sr} /{ }^{86} \mathrm{Sr}$ ratio and $1 / \mathrm{Sr}$ in the Guijiang River. The ${ }^{87} \mathrm{Sr} /{ }^{86} \mathrm{Sr}$ ratios in the mainstream range from 0.710558 to 0.716453 and exhibit an obvious variation trend (Fig. 7). The high values of the headwater samples S1 and S3 (0.715849 and 0.715973, respectively) can be attributed to granites in the Miaoershan Mountain with extremely high ${ }^{87} \mathrm{Sr} /{ }^{86} \mathrm{Sr}$ ratios (Table 4 ). With an abundant exposure of carbonate rocks, import of fertilizers, urban runoff, and municipal water with low $\mathrm{Sr}$ isotope values (Tables 4 and 5), the ${ }^{87} \mathrm{Sr} /{ }^{86} \mathrm{Sr}$ ratios obviously decrease downwards from the source area. The sample S5 has the lowest ${ }^{87} \mathrm{Sr} /{ }^{86} \mathrm{Sr}$ ratio in the Guijiang River because the $\mathrm{Sr}$ isotopic compositions of the Carboniferous carbonate rocks are the lowest in the Late Paleozoic (Huang, 1997). The mainstream ${ }^{87} \mathrm{Sr} /{ }^{86} \mathrm{Sr}$ ratios rise remarkably from samples $\mathrm{S} 5$ to $\mathrm{S} 10$, gradually decrease from S12 to S15, and then fluctuate from S17 to S22. The variation trend is inconsistent with the ${ }^{87} \mathrm{Sr} /{ }^{86} \mathrm{Sr}$ ratios of the tributaries and therefore does not result from the confluence of these tributaries. Previous studies on karst water showed that the water draining sand rocks had the highest ${ }^{87} \mathrm{Sr} /{ }^{86} \mathrm{Sr}$ ratios, followed by dolomites and limestones (Wang and Wang, 2005). Therefore, the variation in the ${ }^{87} \mathrm{Sr} /{ }^{86} \mathrm{Sr}$ ratios in these reaches may be attributed to the subtle differences of the underlying bedrock and/or to anthropogenic influence. After flowing through Pingle County, the ${ }^{87} \mathrm{Sr} /{ }^{86} \mathrm{Sr}$ ratios progressively increase and reach the highest value near the river mouth. The Guijiang River catchment downwards from Pingle flows across Cambrian strata consisting of carbonate rocks intercalated by sand rocks, mud rocks, and shales, which have higher ${ }^{87} \mathrm{Sr} /{ }^{86} \mathrm{Sr}$ ratios than those of the Devonian and Carboniferous rocks (Burke et al., 1982; Huang, 1997). Considering the low ${ }^{87} \mathrm{Sr} /{ }^{86} \mathrm{Sr}$ ratios of carbonate rocks, vegetation, fertilizers, and industrial and domestic wastewater, the high $\mathrm{Sr}$ isotopic values of the
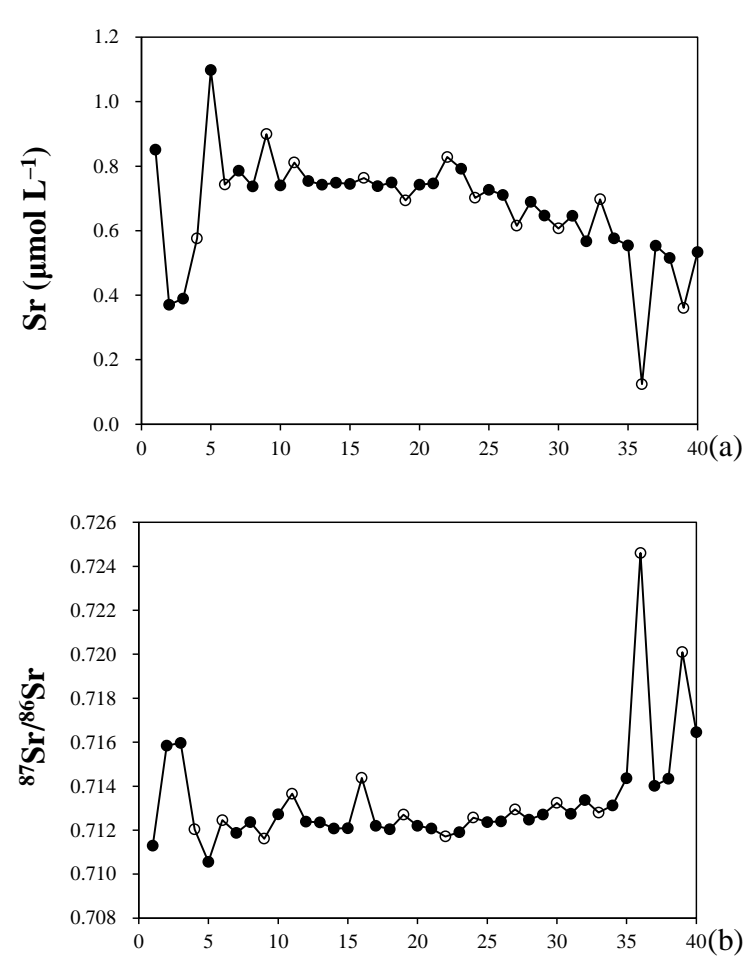

Fig. 7. Spatial variations of the $\mathrm{Sr}$ concentrations (a) and the ${ }^{87} \mathrm{Sr} /{ }^{86} \mathrm{Sr}$ ratios (b) in the Guijiang River. The filled and open circles represent the samples in the mainstreams and tributaries, respectively.

Guijiang River water should be caused by silicate weathering, which is consistent with the high ${ }^{87} \mathrm{Sr} /{ }^{86} \mathrm{Sr}$ ratios of the silicate fraction of the riverbed sediment and soil in the catchment. The tributaries Longjiang River and Siliang River have the highest ${ }^{87} \mathrm{Sr} /{ }^{86} \mathrm{Sr}$ ratios in the entire Guijiang River. As the two tributaries are small enough, the silicate components exposed in a scattered manner can significantly influence the ${ }^{87} \mathrm{Sr} /{ }^{86} \mathrm{Sr}$ ratios of the river water.

Generally, the ${ }^{87} \mathrm{Sr} /{ }^{86} \mathrm{Sr}$ ratios of the Guijiang River are much higher than those of the upper and middle Pearl River (the Nanpan River of 0.70740 to 0.70856 , Xu and Liu, 2007; the Xijiang River of 0.70837 to 0.71049 , Wang et al., 2009 and 0.708487 to 0.710336 , Wei et al., 2013). These ratios are also higher than those of the tributaries Wujiang River (0.707722 to 0.711037) and Yuanjiang River in the karst region (0.708711 to 0.714479$)$ of the Yangtze River (Han and Liu, 2004). This result suggests that although the Guijiang River is a karst river, a few silicate components, such as granites, sand rocks, mud rocks, and shales, with high $\mathrm{Sr}$ isotopic compositions contribute significantly to the ${ }^{87} \mathrm{Sr} /{ }^{86} \mathrm{Sr}$ ratios of the river water. 


\subsection{Implication for studies of the $\mathrm{Sr}$ isotope evolution of seawater}

The significance of the rivers for variations in the seawater ${ }^{87} \mathrm{Sr} /{ }^{86} \mathrm{Sr}$ ratios mainly depends on both the $\mathrm{Sr}$ fluxes and their ${ }^{87} \mathrm{Sr} /{ }^{86} \mathrm{Sr}$ ratios. This significance can be assessed by calculating an "excess ${ }^{87} \mathrm{Sr}$ flux" $\left({ }^{87} \mathrm{Sr}_{\mathrm{ex}}\right.$ ) (Bickle et al., 2003):

${ }^{87} \mathrm{Sr}_{\mathrm{ex}}=\left({ }^{87} \mathrm{Sr} /{ }^{86} \mathrm{Sr}-0.70916\right) \times \mathrm{Sr}_{\text {flux }}$,

where $\mathrm{Sr}_{\text {flux }}$ indicates $\mathrm{Sr}$ fluxes transported by rivers and 0.70916 represents the ${ }^{87} \mathrm{Sr} /{ }^{86} \mathrm{Sr}$ ratio in modern seawater (Hodell et al., 1990). The ${ }^{87} \mathrm{Sr}_{\text {ex }}$ of the Xishui River at Lanxi is $-0.29 \times 10^{3} \mathrm{~mol} \mathrm{yr}^{-1}$ and $-1.6 \times 10^{3} \mathrm{~mol} \mathrm{yr}^{-1}$ in the winter and summer, respectively. The negative values indicate that the Xishui River will decrease the $\mathrm{Sr}$ isotopic compositions of seawater as a silicate watershed. Certainly, only the Xishui River has no visible influence on the $\mathrm{Sr}$ isotope evolution of seawater as a small watershed. However, many rivers draining young basalts also have low ${ }^{87} \mathrm{Sr} /{ }^{86} \mathrm{Sr}$ ratios and cannot contribute to noticeable increases in the $\mathrm{Sr}$ isotopic composition of seawater. For example, Yale and Carpenter (1996) observed a correlation between the formation of large basalt provinces and decreases in the $\mathrm{Sr}$ isotope ratio of the ocean. Taylor and Lasaga (1999) studied the contribution of chemical weathering of the young Columbia basalts to the $\mathrm{Sr}$ isotope evolution of seawater. These authors concluded that sharp decreases in the marine ${ }^{87} \mathrm{Sr} /{ }^{86} \mathrm{Sr}$ ratios reflected periods of increased global weathering rates, and young and old lithological variations could be among the major controlling factors of the marine $\mathrm{Sr}$ isotope record. Allègre et al. (2010) proposed that intensive weathering on volcanic islands, island arcs, and oceanic islands was the missing source of the mantle-derived ${ }^{87} \mathrm{Sr} /{ }^{86} \mathrm{Sr}$ ratio $(0.703)$ in the seawater $\mathrm{Sr}$ isotope balance and represented approximately $60 \%$ of the actual mantle-like input of Sr to the oceans. Therefore, considering that many rivers draining basalt province globally, this type of silicate watershed with low ${ }^{87} \mathrm{Sr} /{ }^{86} \mathrm{Sr}$ ratios may significantly influence the $\mathrm{Sr}$ isotope evolution of seawater. Additionally, many recent studies have shown that the global flux of $\mathrm{CO}_{2}$ consumed by the chemical weathering of basalts represented 30 to $35 \%$ of the consumption flux of the continental silicate (e.g., Gaillardet et al., 1999; Dessert et al., 2003; Dupré et al., 2003). In this case, river catchments with high silicate weathering rates do not transport highly radiogenic Sr into the oceans. Therefore, the use of the $\mathrm{Sr}$ isotope ratio variations of seawater to deduce the continental silicate weathering intensity may be questionable

\section{Conclusions}

As a small silicate watershed, the Xishui River has relatively high $\mathrm{Sr}$ concentrations and low ${ }^{87} \mathrm{Sr} /{ }^{86} \mathrm{Sr}$ ratios. An important reason for these characteristics is that the gneisses and granites that are widely distributed in the catchment have rather low Sr isotope ratios. The ${ }^{87} \mathrm{Sr}_{\mathrm{ex}}$ of the Xishui River at Lanxi is $-0.29 \times 10^{3} \mathrm{~mol} \mathrm{yr}^{-1}$ and $-1.6 \times 10^{3} \mathrm{~mol} \mathrm{yr}^{-1}$ in the winter and summer, respectively, indicating that the Xishui River decreases the $\mathrm{Sr}$ isotope values of seawater. Considering the low ${ }^{87} \mathrm{Sr} /{ }^{86} \mathrm{Sr}$ ratios of young basalts, these rocks may also reduce the seawater $\mathrm{Sr}$ isotope ratios. However, silicate weathering rates in these river catchments are very high and thus significantly affect the atmospheric $\mathrm{CO}_{2}$ consumption and the global climate change. In this sense, there is no direct relationship between the silicate weathering intensity and the sea water $\mathrm{Sr}$ isotope evolution. In contrast, the Guijiang River has low $\mathrm{Sr}$ concentrations and high ${ }^{87} \mathrm{Sr} /{ }^{86} \mathrm{Sr}$ ratios as a small carbonate watershed, which is mainly attributed to the weathering of the exposed silicate components in the catchment.

Acknowledgements. This study was supported by the China Geological Survey Projects (grant no. 12120113005400), the 863 Projects (grant no. 2009AA06Z112), and the Natural Science Foundation of China (project no. 40830107, 40873001 and 41003001). We thank Zhou Bin, Luo Chao, Zhang Qiang, Zhang Chunlai, as well as others for their help in the field and Pu Wei in the laboratory.

Edited by: A. D. Reeves

\section{References}

Allègre, C. J., Louvat, P., Gaillardet, J., Meynadier, L., Rad, S., and Capmas, F.: The fundamental role of island arc weathering in the oceanic Sr isotope budget, Earth Planet. Sc. Lett., 292, 51-56, 2010.

Bickle, M. J., Harris, N. B. W., Bunbury, J., Chapman, H. J., Fairchild, I. J., and Ahmad, T.: Controls on the ${ }^{87} \mathrm{Sr} /{ }^{86} \mathrm{Sr}$ of carbonates in the Garwal Himalaya, headwaters of the Ganges, J. Geol., 109, 737-753, 2001.

Bickle, M. J., Bunbury, J., Chapman, H. J., Harris, N. B. W., Fairchild, I. J., and Ahmad, T.: Fluxes of Sr into the headwaters of the Ganges, Geochim. Cosmochim. Acta, 67, 2567-2584, 2003.

Bickle, M. J., Chapman, H. J., Bunbury, J., Harris, N. B. W., Fairchild, I. J., Ahmad, T., and Pomiès, C.: Relative contributions of silicate and carbonate rocks to riverine $\mathrm{Sr}$ fluxes in the headwaters of the Ganges, Geochim. Cosmochim. Acta, 69, 2221-2240, 2005.

Blum, J. D.: The effect of late Cenozoic glaciation and tectonic uplift on silicate weathering rates and the marine ${ }^{87} \mathrm{Sr} /{ }^{86} \mathrm{Sr}$ record, in: Tectonic Uplift and Climate Change, edited by: Ruddiman, W. R., Plenum, New York, 260-288, 1997. 
Blum, J. D., Gazis, C. A., Jacobson, A. D., and Chamberlain, C. P.: Carbonate versus silicate weathering in the Raikhot watershed within the High Himalayan Crystalline Series, Geology, 26, 411414, 1998

Brass, G. W.: The variation of the marine ${ }^{87} \mathrm{Sr} /{ }^{86} \mathrm{Sr}$ ratio during Phanerozoic time: Interpretation using a flux model, Geochim. Cosmochim. Acta, 40, 721-730, 1976.

Brenot, A., Baran, N., Petelet-Giraud, E., and Négrel, P.: Interaction between different water bodies in a small catchment in the Paris basin (Brévilles, France), Tracing of multiple $\mathrm{Sr}$ sources through $\mathrm{Sr}$ isotopes coupled with $\mathrm{Mg} / \mathrm{Sr}$ and $\mathrm{Ca} / \mathrm{Sr}$ ratios, Appl. Geochem., 23, 58-75, 2008.

Bureau of Geology and Mineral Resources of Hubei Province: Regional Geology of Hubei Province, Beijing, Geological Publishing House, Beijing, 1990.

Burke, W. H., Denison, R. E., Hetherington, E. A., Koepnick, R. B., Nelson, H. F., and Otto, J. B.: Variation of seawater ${ }^{87} \mathrm{Sr} /{ }^{86} \mathrm{Sr}$ throughout Phanerozoic time, Geology, 10, 516-519, 1982.

Capo, R. C. and DePaolo, D. J.: Seawater strontium isotopic variations from 2.5 million years ago to the present, Science, 249, $51-55,1990$.

Chen, B., Jahn, B. M., and Wei, C. J.: Petrogenesis of Mesozoic granitoids in the Dabie UHP complex, Central China: trace element and Nd-Sr isotope evidence, Lithos, 60, 67-88, 2002.

Chesley, J. T., Quade, J., and Ruiz, J.: The Os and Sr isotopic record of Himalayan paleorivers: Himalayan tectonics and influence on ocean chemistry, Earth Planet. Sc. Lett., 179, 115-124, 2000.

Chetelat, B., Liu, C. Q., Zhao, Z. Q., Wang, Q. L., Li, S. L., and Wang, B. L.: Geochemistry of the dissolved load of the Changjiang Basin rivers: Anthropogenic impacts and chemical weathering, Geochim. Cosmochim. Acta, 72, 4254-4277, 2008.

China Geological Survey: Geological Map of the People's Republic of China 1:2500 000, SinoMaps Press, Beijing, 2004

Dalai, T. K., Krishnaswami, S., and Kumar, A.: $\mathrm{Sr}$ and ${ }^{87} \mathrm{Sr} /{ }^{86} \mathrm{Sr}$ in the Yamuna River System in the Himalaya: Sources, fluxes, and controls on $\mathrm{Sr}$ isotope composition, Geochim. Cosmochim. Acta, 67, 2931-2948, 2003.

DePaolo, D. J.: Detailed record of the Neogene Sr isotopic evolution of seawater from DSDP Site 590B, Geology, 14, 103-106, 1986.

DePaolo, D. J. and Ingram, B. L.: High-Resolution Stratigraphy with Strontium Isotopes, Science, 227, 938-941, 1985.

Derry, L. A. and France-Lanord, C.: Neogene Himalayan weathering history and river ${ }^{87} \mathrm{Sr} /{ }^{86} \mathrm{Sr}$ : impact on the marine $\mathrm{Sr}$ record, Earth Planet. Sc. Lett., 142, 59-74, 1996.

Dessert, C., Dupré, B., Gaillardet, J., François, L. M., and Allègre, C. J.: Basalt weathering laws and the impact of basalt weathering on the global carbon cycle, Chem. Geol., 202, 257-273, 2003.

Dupré, B., Dessert, C., Oliva, P., Goddéris, Y., Viers, J., François, L., Millot, R., and Gaillardet, J.: Rivers, chemical weathering and Earth's climate, C. R. Geosci., 335, 1141-1160, 2003.

Edmond, J. M.: Himalayan tectonics, weathering processes, and the strontium isotope record in marine limestones, Science, 258, 1594-1597, 1992.

Edmond, J. M., Palmer, M. R., Measures, C. I., Grant, B., and Stallard, R. F.: Fluvial geochemistry and denudation rate of Guyana Shield, Geochim. Cosmochim. Acta, 59, 3301-3325, 1994.

Elderfield, H. and Gieskes, J. M.: Sr isotopes in interstitial waters of marine sediments from Deep Sea Drilling Project cores, Nature, 300, 493-497, 1982.
English, N. B., Quade, J., DeCelles, P. G., and Garzione, C. N.: Geologic control of $\mathrm{Sr}$ and major element chemistry in Himalayan rivers, Nepal, Geochim. Cosmochim. Acta, 64, 25492566, 2000.

Gaillardet, J., Dupré, B., Louvat, P., and Allègre, C. J.: Global silicate weathering and $\mathrm{CO}_{2}$ consumption rates deduced from the chemistry of large rivers, Chem. Geol., 159, 3-30, 1999.

Galy, A. and France-Lanord, C.: Weathering processes in the Ganges-Brahmaputra basin and the riverine alkalinity budget, Chem. Geol., 159, 31-60, 1999.

Galy, A., France-Lanord, C., and Derry, L. A.: The strontium isotopic budget of Himalayan rivers in Nepal and Bangladesh, Geochim. Cosmochim. Acta, 63, 1905-1925, 1999.

Ge, N. J., Li, H. Y., Hou, Z. H., Bo, L., and Qin, L. P.: Nd-Sr isotope geochemistry of the Baimajian granite in the Dabie Orogen, Geol. Rev., 47, 184-187, 2001a.

Ge, N. J., Li, H. Y., Qin, L. P., Hou, Z. H., and Bo, L.: Sr, Nd and Pb isotope geochemistry of granulites and TTG gneisses from the North Dabie Mountains, Acta Geol. Sin., 75, 379-384, 2001 b.

Gu, S. Y., Hua, R. M., and Qi, H. W.: Study on zircon LA-ICPMS U-Pb dating and $\mathrm{Sr}-\mathrm{Nd}$ isotope of the Guposhan granite in Guangxi, Acta Geol. Sin., 80, 543-553, 2006.

Han, G. L. and Liu, C. Q.: Water geochemistry controlled by carbonate dissolution: a study of the river waters draining karstdominated terrain, Guizhou Province, China, Chem. Geol., 204, $1-21,2004$.

Han, G. L., Tang, Y., and Xu, Z. F.: Fluvial geochemistry of rivers draining karst terrain in Southwest China, J. Asian Earth Sci., 38 , $65-75,2010$

Harris, N. B. W.: Significance of weathering Himalayan metasedimentary rocks and leucogranites for the Sr-isotope evolution of seawater, Geology, 23, 795-798, 1995.

Harris, N. B. W., Bickle, M., Chapman, H., Fairchild, I., and Bunbury, J.: The significance of Himalayan rivers for silicate weathering rates: Evidence from the Bhote Kosi tributary, Chem. Geol., 144, 205-220, 1998.

Hess, J., Bender, M. L., and Schilling, J. G.: Evolution of the ratio of strontium- 87 to strontium-86 in seawater from Cretaceous to present, Science, 231, 979-984, 1986.

Hodell, D. A., Mead, G. A., and Mueller, P. A.: Variation in the strontium isotopic composition of seawater (8 Ma to present) implications for chemical weathering rates and dissolved fluxes to the oceans, Chem. Geol., 80, 291-307, 1990.

Hodell, D. A., Mueller, P. A., and Garrido, J. R.: Variations in the strontium isotopic composition of seawater during the Neogene, Geology, 19, 24-27, 1991.

Hosono, T., Nakano, T., Igeta, A., Tayasu, I., Tanaka, T., and Yachi, S.: Impact of fertilizer on a small watershed of Lake Biwa, Use of sulfur and strontium isotopes in environmental diagnosis, Sci. Total Environ., 384, 342-354, 2007.

Huang, S. J.: A study on carbon and strontium isotopes of late Paleozoic carbonate rocks in the upper Yangtze platform, Acta Geol. Sin., 71, 45-53, 1997.

Jacobsen, A. D. and Blum, J. D.: Ca/Sr and ${ }^{87} \mathrm{Sr} /{ }^{86} \mathrm{Sr}$ geochemistry of disseminated calcite in Himalayan silicate rocks from Nanga Parbat: Influence on river-water chemistry, Geology, 28, 463-466, 2000. 
Jacobson, A. D., Blum, J. D., Chamberlain, C. P., Poage, M. A., and Sloan, V. F.: $\mathrm{Ca} / \mathrm{Sr}$ and $\mathrm{Sr}$ isotope systematics of a Himalayan glacial chronosequence: Carbonate versus silicate weathering rates as a function of landscape surface age, Geochim. Cosmochim. Acta, 66, 13-27, 2002a.

Jacobsen, A. D., Blum, J. D., and Walter, L. M.: Reconciling the elemental and $\mathrm{Sr}$ isotope composition of Himalayan weathering fluxes: Insights from the carbonate geochemistry of stream waters, Geochim. Cosmochim. Acta, 66, 3417-3429, 2002b.

Jahn, B. M., Wu, F. Y., Lo, C. H., and Tsai, C. H.: Crust-mantle interaction induced by deep subduction of the continental crust: geochemical and $\mathrm{Sr}-\mathrm{Nd}$ isotopic evidence from post-collisional mafic-ultramafic intrusions of the northern Dabie complex, central China, Chem. Geol., 157, 119-146, 1999.

Jiang, Y. J., Wu, Y. X., and Yuan, D. X.: Human impacts on karst groundwater contamination deduced by coupled nitrogen with strontium isotopes in the Nandong underground river system in Yunan, China, Environ. Sci. Technol., 43, 7676-7683, 2009.

Karim, A. and Veizer, J.: Weathering processes in the Indus River Basin: implications from riverine carbon, sulfur, oxygen, and strontium isotopes, Chem. Geol., 170, 153-177, 2000.

Korte, C., Jasper, T., Kozur, H. W., and Veizer, J.: ${ }^{87} \mathrm{Sr} /{ }^{86} \mathrm{Sr}$ record of Permian seawater, Palaegeogr. Palaeocl., 240, 89-107, 2006.

Krishnaswami, S., Trivedi, J. R., Sarin, M. M., Ramesh, R., and Sharma, K. K.: Strontium isotopes and Rubidium in the GangaBrahmaputra river system: Weathering in the Himalaya, fluxes to the Bay of Bengal and contributions to the evolution of oceanic ${ }^{87} \mathrm{Sr} /{ }^{86} \mathrm{Sr}$, Earth Planet. Sc. Lett., 109, 243-253, 1992.

Lang, Y. C., Liu, C. Q., Zhao, Z. Q., Li, S. L., and Han, G. L.: Geochemistry of surface and ground water in Guiyang, China: Water/rock interaction and pollution in a karst hydrological system, Appl. Geochem., 21, 887-903, 2006.

Li, S. G., Nie, Y. H., Hart, S. R., and Zhang, Z. Q.: Interaction between subducted continental crust and the mantle - II. Sr and $\mathrm{Nd}$ isotopic geochemistry of the syncollisional mafic-ultramafic intrusions in Dabie Mountains, Sci. China Ser. D, 41, 632-638, 1998.

Li, X. D., Liu, C. Q., Harue, M., Li, S. L., and Liu, X. L.: The use of environmental isotopic (C, Sr, S) and hydrochemical tracers to characterize anthropogenic effects on karst groundwater quality: A case study of the Shuicheng Basin, SW China, Appl. Geochem., 25, 1924-1936, 2010.

Liu, W. J., Liu, C. Q., Zhao, Z. Q., Li, L. B., Tu, C. L., and Liu, T. Z.: The weathering and soil formation process in karstic area, southwest China: A study on strontium isotope geochemistry of yellow and limestone soil profiles, J. Earth Environ., 2, 331-336, 2011.

Liu, Y. C., Xu, S. T., Li, S. G., Jiang, L. L., Wu, W. P., Chen, G. B., and $\mathrm{Su}, \mathrm{W}$.: Geochemical characteristics, $\mathrm{Sr}-\mathrm{Nd}$ isotopic compositions and tectonic implications of eclogite in the North Dabie, Sci. China Ser. D, 30, 99-107, 2000.

Melezhik, V. A., Pokrovsky, B. G., Fallick, A. E., Kuznetsov, A. B., and Bujakaite, M. I.: Constraints on ${ }^{87} \mathrm{Sr} /{ }^{86} \mathrm{Sr}$ of late Ediacaran seawater: insight from Siberian high-Sr limestones, J. Geol. Soc. Lond., 166, 183-191, 2009.

Millot, R., Gaillardet, J., Dupré, B., and Allègre, C. J.: Northern latitude chemical weathering rates: Clues from the Mackenzie River Basin, Canada, Geochim. Cosmochim. Acta, 67, 13051329, 2003.
Négrel, P. and Deschamps, P.: Natural and anthropogenic budgets of a small watershed in the Massif Central (France): Chemical and strontium isotopic characterization of water and sediments, Aquat. Geochem., 2, 1-27, 1996.

Négrel, P., Allègre, C. J., Dupré, B., and Lewin, E.: Erosion sources determined by inversion of major and trace element ratios and strontium isotopic ratios in river water; the Congo Basin case, Earth Planet. Sc. Lett., 120, 59-76, 1993.

Oliva, P., Viers, J., and Dupré, B.: Chemical weathering in granitic environments, Chem. Geol., 202, 225-256, 2003.

Oliva, P., Dupré, B., Martin, F., and Viers, J.: The role of trace minerals in chemical weathering in a high-elevation granitic watershed (Estibere, France): Chemical and mineralogical evidence, Geochim. Cosmochim. Acta, 68, 2223-2244, 2004.

Palmer, M. R. and Edmond, J. M.: The strontium isotope budget of the modern ocean, Earth Planet. Sc. Lett., 92, 11-26, 1989.

Palmer, M. R. and Edmond, J. M.: Controls over the strontium isotope composition of river water, Geochim. Cosmochim. Acta, 56, 2099-2111, 1992.

Palmer, M. R. and Elderfield, H.: Sr isotope composition of seawater over the past $75 \mathrm{Myr}$, Nature, 314, 526-528, 1985.

Quade, J., Roe, L., DeCelles, P. G., and Ojha, T. P.: The late Neogene ${ }^{87} \mathrm{Sr} /{ }^{86} \mathrm{Sr}$ record of lowland Himalayan rivers, Science, 276, 1828-1831, 1997.

Raymo, M. E., Ruddiman, W. F., and Froelich, P. N.: Influence of Late Cenozoic mountain building on ocean geochemical cycles, Geology, 16, 649-653, 1988.

Richter, F. M. and DePaolo, D. J.: Numerical models for diagenesis and the Neogene $\mathrm{Sr}$ isotopic evolution of seawater from DSDP Site 590B, Earth Planet. Sc. Lett., 83, 27-38, 1987.

Richter, F. M. and DePaolo, D. J.: Diagenesis and Sr isotopic evolution of seawater using data from DSDP 590B and 575, Earth Planet. Sc. Lett., 90, 382-394, 1988.

Richter, F. M., Rowley, D. B., and DePaolo, D. J.: Sr isotope evolution of seawater: the role of tectonics, Earth Planet. Sc. Lett., 109, 11-23, 1992.

Singh, S. K., Trivedi, J. R., Pande, K., Ramesh, R., and Krishnaswami, S.: Chemical and $\mathrm{Sr}, \mathrm{O}, \mathrm{C}$, isotopic compositions of carbonates from the Lesser Himalaya: Implications to the $\mathrm{Sr}$ isotope composition of the source waters of the Ganges, Ghaghara and the Indus Rivers, Geochim. Cosmochim. Acta, 62, 743-755, 1998.

Singh, S. K., Kumar, A., and France-Lanord, C.: $\mathrm{Sr}$ and ${ }^{87} \mathrm{Sr} /{ }^{86} \mathrm{Sr}$ in waters and sediments of the Brahmaputra river system: Silicate weathering, $\mathrm{CO}_{2}$ consumption and $\mathrm{Sr}$ flux, Chem. Geol., 234, 308-320, 2006.

Taylor, A. S. and Lasaga, A. C.: The role of basalt weathering in the $\mathrm{Sr}$ isotope budget of the oceans, Chem. Geol., 161, 199-214, 1999.

Veizer, J.: Strontium isotopes in seawater through time, Ann. Rev. Planet. Sci., 17, 141-167, 1989.

Veizer, J. and Compston, W.: ${ }^{87} \mathrm{Sr} /{ }^{86} \mathrm{Sr}$ composition of seawater during the Phanerozoic, Geochim. Cosmochim. Acta, 38, 14611484, 1974.

Veizer, J., Ala, D., Azmy, K., Bruckschen, P., Buhl, D., Bruhn, F., Carden, G. A. F., Diener, A., Ebneth, S., Godderis, Y., Jasper, T., Korte, C., Pawellek, F., Podlaha, O. G., and Strauss, H.: ${ }^{87} \mathrm{Sr} /{ }^{86} \mathrm{Sr}, \delta^{13} \mathrm{C}$ and $\delta^{18} \mathrm{O}$ evolution of Phanerozoic seawater, Chem. Geol., 161, 59-88, 1999. 
Vitòria, L., Otero, N., Soler, A., and Canals, A.: Fertilizer characterization: isotopic data (N, S, O, C, and Sr), Environ. Sci. Technol., 38, 3254-3262, 2004.

Wang, B., Lee, X. Q., Yuan, H. L., Zhou, H., and Zhao, Y. L.: Geochemical characteristics of main ion and $\mathrm{Sr}$ isotope in the main channel of Xijiang River, South China, Geochimica, 38, 345$353,2009$.

Wang, T. and Wang, Z. Y.: ${ }^{87} \mathrm{Sr} /{ }^{86} \mathrm{Sr}$ characteristics of karst water in Guilin area, Acta Geosci. Sin., 26 (supplement), 299-302, 2005.

Wang, Y. J., Fan, W. M., Peng, T. P., Zhang, H. F., and Guo, F.: Nature of the Mesozoic lithospheric mantle and tectonic decoupling beneath the Dabie Orogen, Central China: Evidence from ${ }^{40} \mathrm{Ar} /{ }^{39} \mathrm{Ar}$ geochronology, elemental and $\mathrm{Sr}-\mathrm{Nd}-\mathrm{Pb}$ isotopic compositions of early Cretaceous mafic igneous rocks, Chem. Geol., 220, 165-189, 2005.

Wawrzenitz, N., Romer, R. L., Oberhänsli, R., and Dong, S. W.: Dating of subduction and differential exhumation of UHP rocks from the Central Dabie Complex (E-China): Constraints from microfabrics, $\mathrm{Rb}-\mathrm{Sr}$ and $\mathrm{U}-\mathrm{Pb}$ isotope systems, Lithos, 89, 174201, 2006.

Wei, G. J., Ma, J. L., Liu, Y., Xie, L. H., Deng, W. F., Ren, Z. Y., Zeng, T., and Yang, Y. H.: Seasonal changes in the radiogenic and stable strontium isotopic composition of Xijiang River water: Implications for chemical weathering, Chem. Geol., 343, 67-75, 2013.

White, A. F. and Blum, A. E.: Effects of climate on chemical weathering in watersheds, Geochim. Gosmochim. Acta, 59, 17291747, 1995.

Wu, L. L., Huh, Y., Qin, J. H., Du, G., and Van Der Lee, S.: Chemical weathering in the Upper Huang He (Yellow River) draining the eastern Qinghai-Tibet Plateau, Geochim. Cosmochim. Acta, 69, 5279-5294, 2005.

Wu, W. H., Zheng, H. B., Yang, J. D., Luo, C., and Zhou, B.: Chemical weathering, atmospheric $\mathrm{CO}_{2}$ consumption, and the controlling factors of small silicate watershed in subtropical zone: implications for the global carbon cycle research, Chem. Geol., 356, 141-150, 2013.

$\mathrm{Xu}$, W. C. and Zhang, Y. H.: Study on strontium, oxygen, neodymium and lead isotopes of Mt. Miaoershan granite batholith in south China, Guangxi Geol., 6, 15-22, 1993.
Xu, W. C., Zhang, Y. H., and Liu, Y. B.: Progression in geochronological study and scheme of chronoclassfication on Miaoershan granite batholith, Acta Petrol. Sin., 10, 330-337, 1994.

$\mathrm{Xu}, \mathrm{Z}$. F. and Liu, C. Q.: Chemical weathering in the upper reaches of Xijiang River draining the Yunnan-Guizhou Plateau, Southwest China, Chem. Geol., 239, 83-95, 2007.

Yale, L. B. and Carpenter, S. J.: Modeling the effects of large igneous provinces on the ${ }^{87} \mathrm{Sr} /{ }^{86} \mathrm{Sr}$ ratio of seawater, GSA Abst. Prog. A, 28, 428, 1996.

Zeng, Y. J., Huang, S. J., Yi, T. F., Hao, X. F., Xiong, C. L., and Hu, Z. W.: Sr isotopic characteristics of carbonate rocks in Xinduqiao formation of Xikang group, western Sichuan, China, J. Earth Sci. Environ., 29, 126-129, 2007.

Zhang, F. F., Wang, Y. J., Zhang, A. M., Fan, W. M., Zhang, Y. Z., and Zi, J. W.: Geochronological and geochemical constraints on the petrogenesis of middle Paleozoic (Kwangsian) massive granites in the eastern south China block, Lithos, 150, 188-208, 2012.

Zhang, H. B., He, S. Y., Yu, S., Wang, Y. X., and Wang, L. L.: Hydrochemical characteristics and influencing factors of the river water in the Guijiang, Carsologica Sinica, 31, 395-401, 2012.

Zhang, H. F., Gao, S., Zhong, Z. Q., Zhang, B. R., Zhang, L., and $\mathrm{Hu}, \mathrm{S}$. H.: Geochemical and $\mathrm{Sr}-\mathrm{Nd}-\mathrm{Pb}$ isotopic compositions of Cretaceous granitoids: constraints on tectonic framework and crustal structure of the Dabieshan ultrahigh-pressure metamorphic belt, China, Chem. Geol., 186, 281-299, 2002.

Zheng, H. Y., Liu, C. Q., Wang, Z. L., Yang, C., Chen, S., and Zhu, S. F.: Strontium isotopes as a tracer of plant nutrition element source in yellow soil region of Guizhou Province, J. Beijing Forest. Univ., 30, 72-76, 2008.

Zheng, X. S., Jin, C. W., Zhai, M. G., and Shi, Y. H.: Approach to the source of the gray gneisses in North Dabie terrain: Sm$\mathrm{Nd}$ isochron age and isotope composition, Acta Petrol. Sin., 16, 194-198, 2000.

Zhu, J. C., Li, X. D., Shen, W. Z., Wang, Y. X., and Yang, J. D.: Sr, $\mathrm{Nd}$ and $\mathrm{O}$ isotope studies on the genesis of the Huashan granite complex, Acta Geol. Sin., 3, 225-235, 1989.

Zhu, X. L., Wang, S. J., and Luo, W. J.: Characteristics of strontium isotopes and their implications in the Qixing Cave of Guizhou, China, Chinese Sci. Bull., 56, 670-675, 2011. 